Jacek Makowski

\title{
Von IMAGEPflege ZUR DisKREDITIERUNG POLITISCHER \\ Gegner. Hassrede als Strategie \\ VON ONLINE-PRESSEMITTEILUNGEN
}

\section{Einleitung}

Dem Vorspann der auf der Webseite der Alternative für Deutschland veröffentlichten Pressemitteilung vom 16. Februar 2018 ist das folgende Statement zu entnehmen:

Die zerstrittene und in der Wählergunst abstürzende SPD wird bald mehr Parteimitglieder, als Wähler haben und bei der CSU symbolisiert der Buchstabe "C" inzwischen die islamische Halbmond-Sichel anstelle des Christentums (T43, vgl. Anhang in diesem Beitrag).

Der in dem Leadsatz angekündigte Ton wird im weiteren Verlauf des Textes noch zugespitzt und mündet in Ausdrücken wie Groko-Gruselkoalition, rote Freak-Show, sadistische Domina oder personifizierte Schlaftablette (T43) als Bezeichnungen für gegnerische Fraktionen und deren Vertreter ${ }^{1}$. Es stellt sich die offensichtliche Frage, inwieweit eine derart abwertende Ausdrucksweise gegenüber der politischen Konkurrenz ihren Weg in eines der zentralen Kommunikationsinstrumente der Public Relations finden konnte, das der einschlägigen Literatur zufolge in erster Linie ein Informationsangebot über den Textproduzenten enthalten und stilistisch den gängigen journalistischen Darstellungsformen nachempfunden sein solle.

Der vorliegende Beitrag versteht sich als erster Schritt zu einer Pilotstudie, deren Gegenstand der Einsatz von Online-Pressemitteilung im politischen Marketing bildet. Die wesentliche Fragestellung richtet sich

\footnotetext{
${ }^{1}$ Aus stilistischen Gründen wird auf Doppelformen wie Vertreterinnen und Vertreter verzichtet. Falls nicht gesondert vermerkt, beziehen sich Bezeichnungen wie Bürger gleichermaßen auf Bürger und Bürgerinnen.
} 
dabei auf der belegbaren pejorativierenden, diskreditierenden und abwertenden Strategien sowie dem Einsatz von verschiedenen Erscheinungsformen von Hassrede 2 in diesem aus seiner klassischen Form für das Web 2.0 ${ }^{3}$ übernommenen Instrument der Public Relations, was eine offensichtliche Abweichung von der prototypischen informierend-profilierenden Funktion dieser Textsorten bildet.

Hierbei wird von der These ausgegangen, dass sich der Einsatz von Erscheinungsformen von Hassrede als Strategie zur Diskreditierung politischer Gegner versteht. Wenn dabei abwertende Sprachhandlungen quasi unter dem Deckmantel einer Textsorte realisiert werden, deren primäres Ziel die auf den Textemittenten bezogene Information und Profilierung darstellt, handelt es sich in solchen Fällen um einen klaren Fall von einer vorsätzlichen, sprachmanipulativen Strategie.

Vor dem Hintergrund eines Überblicks über die Charakteristik des politischen Marketings sowie der Zielhierarchie politischer Textsorten erfolgt in diesem Beitrag die Beschreibung der Online-Pressemitteilung als einer Textsorte in politischem Sprachgebrauch. Erörtert werden dabei die Eigenschaften der Textsorte Pressemitteilung in deren ursprünglichen sowie digitalen Form wie auch die in der einschlägigen Literatur vorgenommenen Typologisierungsversuche von Textsorten im politischen Sprachgebrauch. Anschließend wird in Anlehnung an die erarbeiteten Erkenntnisse das Untersuchungskorpus der geplanten Pilotstudie dargelegt sowie eine textsortenorientierte Analyse eines Textbeispiels vorgenommen.

\section{Politisches Marketing - von Imagepflege zur Diskreditierung politischer Gegner}

Wechselwähler und Verbraucher statt Anhängern und Stammwählern, Politik als Warenmarkt und Parteien als Anbieter und Dienstleistungsunternehmen: so in etwa präsentiert sich das von Klein (vgl. 1996, 2016) aufgezeichnete moderne Marktmodell in der politischen Kommunikation. Ausgehend von der Annahme, dass Kommunikation mit Öffentlichkeiten außer der Darstellung eigener Positionen meist auch das Ziel verfolgt,

\footnotetext{
${ }^{2}$ Im vorliegenden Beitrag wird die Bezeichnung Hassrede nach der Auffassung von Meibauer (2013) verstanden, nämlich als ,der sprachliche Ausdruck von Hass gegen Personen oder Gruppen“, im Besonderen „durch die Verwendung von Ausdrücken, die der Herabsetzung und Verunglimpfung von Bevölkerungsgruppen dienen“ (Meibauer 2013: 1).

${ }^{3}$ In naheliegender Zukunft im Hinblick auf die Internetforschung diskutierten Entwicklungsprognosen wohl auch über das Web 3.0 bis hin zum Web 4.0, vgl. hierzu etwa Siever/Schlobinski 2012.
} 
„möglichst viele Mitglieder dieser Öffentlichkeiten von der eigenen Position zu überzeugen" (vgl. Reissen-Kosch 2016: 1), kann in Anlehnung an Dieckmann (1975: 27) die Auseinandersetzung mit den appellativen Elementen verschiedener Kommunikationsbereiche wie etwa im politischen, wirtschaftlichen oder religiösen Sprachgebrauch unter dem Oberbegriff der überredenden Sprache (persuasive language) ${ }^{4}$ erfolgen (vgl. Dieckmann 1976: 27). Aus marktstrategischer Perspektive versteht sich Marketing insbesondere als „die unternehmerische Aufgabe, Marktveränderungen und Bedürfnisverschiebungen sowie Möglichkeiten zur Steigerung des Kundennutzens zu erkennen, um Wettbewerbsvorteile aufbauen zu können" (Gabler 2010: 290). Zur erfolgreichen Marktpositionierung gegenüber von Konkurrenzunternehmen werden insbesondere marketingpolitische Instrumente eingesetzt wie Produkt- und Programmpolitik, Preis- und Konditionenpolitik, Distributionspolitik und Kommunikationspolitik (vgl. Gabler 2010: 289-290). Unter der letzteren versteht Janich (2010) Instrumente wie Werbung, persönlichen Verkauf oder Direktmarketing, Verkaufsförderung und Public Relations, mit denen „Informationen über das Produkt kommuniziert werden" (Janich 2010: 22). Einzelne Marktinstrumente, so Janich, stehen im sogenannten „Marketing-Mix in funktionaler und zeitlicher Beziehung zueinander" (Janich 2010: 22), ergo einer „optimale[n] Kombination der zeitraum- und markt- bzw. marktsegmentbezogen eingesetzten marketingpolitischen Instrumente [...], um die verfolgten Marketingziele zu erreichen" (Gabler 2010: 290).

Im Zuge von Erscheinungen wie „Individualisierung“, „Wertewandel“, Entideologisierung“ und „Stimmungsdemokratie“ (vgl. Klein 1996: 5) erfolgte nach den ersten zwei Jahrzehnten der Bundesrepublik Deutschland das Ablösen des sogenannten Anhängerschaftsmodells durch die Dominanz des Marktmodells in der politischen Kommunikation (vgl. Klein 1996: 5 und 2016: 104). Während in dem Anhängerschaftsmodell die Parteien als „Weltanschauungs- und Milieuparteien“ fungierten (Klein 1996: 5), der Großteil der Bürger eine feste Parteibindung hatte und die politische Konkurrenz als „der angestammte Gegner“ und der „verhasste Feind“ aufgefasst wurde (vgl. Klein 2016: 104), versteht sich im derzeit

\footnotetext{
4 Auf die umfangreichen und oft kontrovers beurteilten terminologischen Abgrenzungsversuche, zuallererst des hier von Dieckmann (1976) konzipierten Konfliktfelds zwischen Überzeugen und Überreden, ferner der damit einhergehenden Phänomene wie Werbung, Public Relations (PR), Persuasion, Manipulation oder Propaganda kann im Hinblick auf den begrenzten Rahmen dieses Beitrags nicht im Detail eingegangen werden, vgl. hierzu u. a. Dieckmann 1976; Mikołajczyk 2004; Ortak 2004; Merten 2008; Janich 2010; Makowski 2011a, 2011b und 2013; Merten 2008 oder Reissen-Kosch 2016.
} 
dominierenden Marktmodell politischer Kommunikation die Politik als „ein funktionierender Markt mit Kunden, die sich nüchtern das politische Angebot anschauen und dann wählen", der moderne Wählertyp als Verbraucher mit bestenfalls leichten Parteipräferenzen und die Parteien als Anbieter (vgl. Klein 2016: 104). Zu verwischen scheint sich dabei die einst im Anhängerschaftsmodell zwischen der „eigenen »politischen Heimat« und den Kräften, die diese bedrohten "verlaufende charakteristische Linie für „Dialogblockaden“, im modernen Marktmodell wird diese vielmehr zwischen „den Bürgern“ und der „Kaste der Politiker“ gezogen (vgl. Klein 1996: 5). Im Hinblick auf die Kommerzialisierung politischen Marketings, in der einige Autoren zugleich als Folge der Globalisierung und Medialisierung auch eine Amerikanisierung der politischen Kommunikation sehen ${ }^{5}$, stellt sich die Frage, ,inwieweit mit medienbedingtem politischem Stilwandel nicht auch ein Legitimitätswandel in der Politik einhergeht, schleichend, manchmal spektakulär, oft aber unspektakulär durch allmähliche Anpassung an moderne Präsentationslogiken und Wahrnehmungsmuster" (Sarcinelli 2009: 112), mit Medien als einer Art „Turboloader“, Medium und Faktor der Veränderung zugleich. Gemeint sei damit wohl eine „Pluralisierung, Individualisierung und Säkularisierung (zumindest im Sinne einer Entkirchlichung), durch die sich die Bindungen an gesellschaftliche und politische Institutionen gelockert haben" (Sarcinelli 2009: 112). In einer Gesellschaft, in der „politisches Verhalten mit abnehmender Tendenz durch Tradition, Milieuzugehörigkeit, weltanschauliche Verankerung, Organisationstreue und stabiles Institutionenvertrauen bestimmt und mehr und mehr zu einem Verhalten der $» W a h l «, d$. h. des »rationalen« Kalküls im Sinne individueller Nutzenorientierung wird, steigen die Chancen für kurzfristige kommunikative Einflüsse" (Sarcinelli 2009: 112).

Auf parallelen zwischen wirtschaftlicher und politischer Werbung bei gleichzeitiger Zuspitzung der Expressivität des politischen Stils und emotionaler Schwerpunktsetzung auch zunehmend außerhalb der Wahlkampfzeiten als bewährter Strategie der Wirtschaftswerbung wird mehrfach in der einschlägigen Literatur verwiesen (vgl. Roth 1987: 298; Kießling/Zolleis 2005: 47; Sarcinelli 2005: 100 oder 2009: 102 und Reissen-Kosch 2016: 7-11). Der Grund hierfür liege unter anderem in dem „wachsenden Druck, ständig präsent zu sein“ (Reissen-Kosch 2016: 10), durch die „Ausweitung des Mediensystems, die Zunahme der Anbieter

${ }^{5}$ Sarcinelli (2005) verweist in diesem Kontext auf die einhergehende „Abkehr von der Meinungsvermittlung hin zu aufmerksamkeitsstarken Marketingaktionen”, vgl. Sarcinelli 2005: 200ff., bei Reissen-Kosch 2016: 10. 
und die wachsende Unübersichtlichkeit der Angebote" geraten politische Funktionsträger auf dem modernen Medienmarkt zunehmend unter „Professionalisierungsdruck, wenn es darum geht, mediale Aufmerksamkeit zu erzeugen" (Sarcinelli 2009: 180).

Eine für den vorliegenden Beitrag wesentliche Parallele zwischen dem wirtschaftlichen und politischen Kommunikationsbereich ergibt sich aus der Zusammenstellung der durch die Werbewirtschaft unterschiedenen verschiedenen Werbeziele (vgl. Janich 2010: 25) und der Übersicht über die Grundfunktionen, Teilziele und Handlungsmuster in politischen Textsorten im Hinblick auf das Kriterium der Imagepflege (Tillmann 1989: 245-278). Janich (2010) verweist zum einen auf die Klassifikation der Werbeziele nach Schweiger/Schrattenecker (1995: 55) in Einführungs-, Erhaltungs- oder Erinnerungswerbung, Stabilisierungs- und Expansionswerbung sowie Imagebildung, geht aber auch auf eine alternative Unterscheidung der Werbestrategien nach Bruhn (2004: 212) in Bekanntmachungs-, Informations-, Imageprofilierungs-, Konkurrenzabgrenzungs-, Zielgruppenerschließungs- und Kontaktanbahnungsstrategie ein, wo die Imagebildung als „übergreifendes Werbeziel [...] je nach Marktsituation [...] meist zugleich der Erhaltung oder Stabilisierung dient" (Schweiger/Schrattenecker 1995: 55, vgl. Janich 2010: 25). Imagewerbung sei dabei als Texthandlung etwa im Sinn von „das werbende Unternehmen positiv vorstellen" (vgl. Janich 2010: 123) aufzufassen. Im Zusammenhang mit der Zulassung von vergleichender Werbung in Deutschland verweist Janich (2010: 123) aber auch auf das fakultative Auftreten der Texthandlung „ein anderes Produkt durch Vergleich in negatives Licht rücken“.

Bei der Aufstellung der politischen Zielhierarchien im politischen Kommunikationsbereich zur Klassifizierung genuiner politischer Textsorten sieht Tillmann (1989) im politischen Sprachgebrauch für den politisch Handelnden das Hauptziel in erster Linie darin, „sich im Bewußtsein der Wahlbürger einseitig kommunikative Vorteile gegenüber dem politischen Gegner zu verschaffen" (Tillmann 1989: 122). Diesem Zweck dienen wiederum zwei konträre kommunikativ zu realisierende Teilziele - Imagepflege (Aufwertung) vs. Diskreditierung politischer Konkurrenz (Abwertung) 6 , welche durch weitere Unterziele und Strategien realisiert werden, die der Komplexität politischen Handelns gerecht werden müssen und nicht selten zumeist gleichzeitig verfolgt werden. Somit erfolgt

\footnotetext{
${ }^{6}$ Bei seiner Aufteilung in die vorliegenden Teilziele verweist Tillmann (1989: 122) auf die bei Zimmermann (1969: 18f.) in diesem Zusammenhang verwendeten Bezeichnungen Aufwertung und Abwertung.
} 
die Durchsetzung der eigenen Imagepflege im Sinn einer Aufwertungs-, Legitimations- und Werbefunktion durch die Profilierungs- und die Polarisierungsstrategie, welche durch Strategien wie den Aufweis der eigenen Legitimation und der Legitimation der Partei/Fraktion, Nachweis und Hervorhebung eigener Wissens- und/oder Wollenskompetenz oder den Aufweis unterschiedlicher Wert- und Zielvorstellungen sowie inhaltlicher Gegensätze und In-Aussicht-Stellung positiver Sanktionen realisiert werden sollen. Den Gegenpol zur Imagepflege bildet demnach die Diskreditierung des politischen Gegners mittels Entlarvung und Diskriminierung, zu deren Realisierung Strategien gezählt werden können wie Aufweis der Defizite, Nachweis von Glaubwürdigkeits- und Kompetenzmangel, Handlungsunfähigkeit, Konzeptionslosigkeit oder Fehlverhalten bezüglich der Einschätzung oder Handlung, In-Frage-Stellen der Berechenbarkeit, Bezichtigung der Lüge, Ausüben von Rechtfertigungszwang, In-Zweifel-Ziehen persönlicher Integrität, Geringschätzung des Handelns, Prophezeiung negativer Sanktionen oder Lächerlich-Machen (vgl. Tillmann 1989: 126, 245-278).

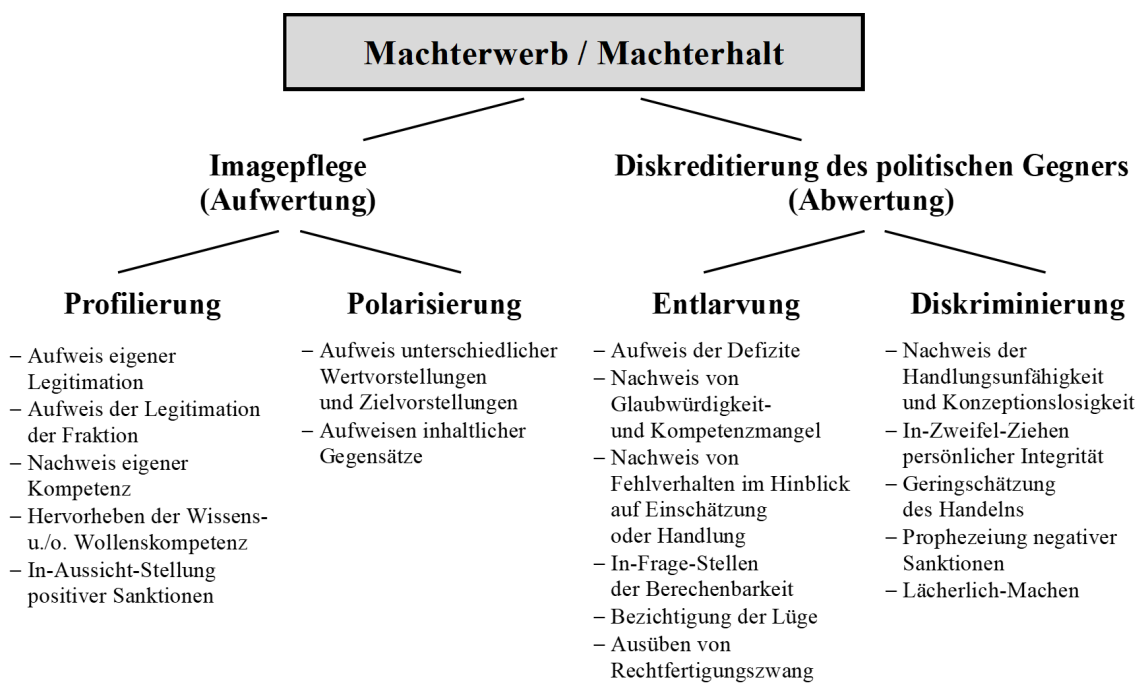

Abb. 1. Übersicht: Grundfunktion, Teilziele und Handlungsmuster in politischen Textsorten

Quelle: Eigene Bearbeitung in Anlehnung an Tillmann 1989: 126, 245-278, vgl. hierzu Makowski 2013: 114-117. 
Aus der Perspektive der Diskussion um das Phänomen von Hassrede im öffentlichen Raum ist an dieser Stelle auf eine dem Anschein nach immer stärkere Disproportion zwischen den Teilzielen Imagepflege und Diskreditierung politischer Gegner im politischen Sprachgebrauch zu verweisen. Während im Fall kommerzieller Werbung auch trotz der von Janich im Hinblick auf die Zulassung von Vergleichswerbung angeführten Texthandlung des In-negatives-Licht-Rückens von Konkurrenzprodukten (vgl. Janich 2010: 123) die informative und profilierende Hauptfunktion stets als stark dominierend aufzufassen ist, scheint im politischen Diskurs sowohl in der Legislaturperiode und besonders zugespitzt im Wahlkampf die Profilierung hauptsächlich durch die Herabsetzung der politischen Konkurrenz etwa im Rahmen negativer Wahlkampagnen, schwarzer PR oder Fake News zu erfolgen.

\section{Online-Pressemitteilung in politischem Sprachgebrauch}

In der einschlägigen Literatur wird die Pressemitteilung7 als das zentrale Kommunikationsinstrument und die Kerntextsorte der Public Relations betrachtet (vgl. u. a. Christoph 2008: 119 und Schweiger/Jungnickel 2011: 400). Als zentrale Textsorte der Medienarbeit ist sie „ein zur Veröffentlichung in den Medien entworfener und herausgegebener Text, der Belange von Unternehmen oder Organisationen kommuniziert" (Schach 2015: 206). Obwohl die Pressemitteilung auch als „das am meisten eingesetzte Standardmittel der Public Relations" bezeichnet wird (Deg 2017: 69), existieren im Fall von Pressemitteilungen, etwa im Gegensatz zur DIN 5008 für Geschäftsbriefe, keine allgemeinen verbindlichen Richtlinien, vielmehr haben sich die geltenden Vorgaben und Regeln im Hinblick auf Gestaltung und Aufbau in der Praxis herausgebildet (vgl. Bischl 2015: 27). Laut der Ratgeberliteratur (vgl. hierzu u. a. Falkenberg 2006; Wolff 2010; Zehrt 2014; Bischl 2015; Schach 2015; Deg 2017; Franck 2017) sind Pressemitteilungen „schriftliche Mitteilungen an die Presse, deren Veröffentlichung im redaktionellen Teil gewünscht wird“ (Christoph 2008: 119). Ferner soll die Pressemitteilung auf eine Weise verfasst werden, dass „der Text ohne Änderung veröffentlicht werden kann“, sie soll „ein Informationsangebot an die Redaktionen“ sein, „die über Änderungen und Abdruck frei entscheiden“ können und liefert „Informationen aus medienrelevantem

\footnotetext{
${ }^{7}$ Parallel zu der Bezeichnung Pressemitteilung verweist Christoph (2008: 120 und 2009: 84) auf die synonymen Formen Pressenotiz, Presseerklärung, Presseinfo oder Pressekommuniqué.
} 
Anlass über einen Sachverhalt, ein Ereignis oder eine Einschätzung mittels Fakten und/oder Zitaten" (Christoph 2008: 119).

Im Hinblick auf die Makrostruktur richten sich die Pressemitteilungen nach einem mehr oder minder festem Layout, das die nachstehenden obligatorischen und fakultativen Komponenten umfasst:

- Logo des Absenders,

- Textsortenkennzeichnung (durch das Wort Pressemitteilung ggf. einen synonymen Ausdruck),

- Sperrfrist (falls vorhanden),

- Schlagzeile, ggf. begleitet durch Dachzeile und/oder Unterzeile,

- eigentlicher Text, eingeleitet durch Spitzmarke mit Orts- und Zeitangabe,

- Einstieg, Leadsatz oder Vorspann (erster Absatz) mit den sechs W-Fragen (wer?, was?, wann?, wie?, wo?, warum?),

- Hauptteil mit weitergehenden Informationen, Absätze werden evtl. durch Zwischenüberschriften getrennt, wobei der Bedeutungsgrad der Informationen wie etwa im Fall der harten Nachricht in der Regel dem Prinzip der (achronologischen) Wichtigkeitsabstufung (vgl. Greule 2017: 66-67) im Sinn einer Nachrichtenpyramide von Kern- über Quellen- und Detail- bis hin zu Hintergrundinformationen abnimmt (vgl. Zehrt 2014: 59),

- Informationen für den Journalisten, zum Beispiel: Längenangabe, Abbinder oder Boilerplate (Kurzporträt des Absenderunternehmens), Kontaktdaten und Ansprechpartner, Bildmaterial, Links oder Verweise auf weitere Informationen, Bildmaterial, andere Texte, Downloadmöglichkeiten etc. (vgl. Christoph 2008: 120-122; Bischl 2015: 27-28; Schach 2015: 210-211).

Per se ist eine Pressemitteilung im Nachrichtenstil verfasst, da sie als „schriftliche Mitteilung an die Redaktion von Presse und Rundfunk, die nach journalistischen Kriterien aufbereitet ist und je nach Zielgruppe unterschiedlich formuliert wird“ (Merten 2000: 240), stilistisch den gängigen journalistischen Darstellungsformen folgt (vgl. Christoph 2009: 84). Die häufigste Form sei dabei die Pressemeldung (vgl. Christoph 2009: 84), allerdings wird auch auf Pressemitteilungen auf der Vorlage von harten (Hard News) oder weichen Nachrichten (Soft News), PR-Meldungen, Personalmeldungen (Personalia), harten (Sachberichten) oder bunten (aufgetauten) Berichten mit Feature-Elementen oder Features hingewiesen (vgl. Bischl 2015: 81-101). Auf diesen Bezug auf journalistische Textsorten lassen sich auch die wesentlichen Charakteristika der Textsorte Pressemitteilung zurückführen: sachlicher, informativer Stil, ein Thema mit Nachrichtenwert, 
deskriptive Themenentfaltung sowie ein Aufbau aus Überschrift, Lead mit den wesentlichsten W-Fragen sowie Hauptteil (vgl. Christoph 2008: 131). Andererseits ist als eines der ausschlaggebenden textexternen Merkmale der Textsorte Pressemitteilung der Umstand aufzufassen, dass der Textemittent eine Art „Schnittmenge mit dem Textgegenstand bildet“, was auf einen deutlich ausgeprägten Eigenwerbungscharakter sowie den Einsatz von Strategien und persuasiven sprachlichen Mitteln schließen lässt, um „eine positive Meinung der Rezipienten gegenüber dem dargestellten Textgegenstand - und damit gegenüber dem Emittenten, also sich selbst herzustellen" (vgl. hierzu Hagl 2011: 72).

Bei ihrem Klassifikations- und Beschreibungsversuch der Textsorte Pressemitteilung primär innerhalb des Kommunikationsbereichs der Wirtschaft ${ }^{8}$ verweist Christoph in diesem Zusammenhang auf eine strukturelle Kopplung der Kommunikationsbereiche der Wirtschaft und des Journalismus innerhalb der Textsorte Pressemitteilung. Somit ist bei der Textfunktion auf der einen Seite aus der Sicht des Textemittenten (Unternehmen, politische Partei, Institution etc.) in der traditionellen Pressemitteilung immer auf eine starke Appellfunktion hinzuweisen, die Pressemitteilung enthält nämlich zunächst die Aufforderung des Emittenten an die Medien, den Text zu publizieren, etwa durch die Textsortenkennzeichnung. Ferner besteht die Appellfunktion in dem an die Medienrezipienten gerichteten Appell, ihre Haltung gegenüber dem Textemittenten - etwa dem Absenderunternehmen - zu verändern, dessen Produkte zu kaufen oder Dienstleistungen in Anspruch zu nehmen. Andererseits ist die Informationsfunktion, der bereits angesprochene Nachrichtenwert von Pressemitteilungen, als ein „Tribut an das System »Journalismus « und dessen Funktion der „Konstruktion von Aktualität“ zu betrachten, bei der Themenwahl wird somit das Interesse des Textemittenten (positive Ereignisse, Erfolgsmeldungen, Expertenwissen) mit Aktualität und Nachrichtenwert ausbalanciert (vgl. Christoph 2008: 132). Auf eine analoge Kopplung ist auch im Hinblick auf die Themenentfaltung zu verweisen: einerseits bilden für die jeder Pressemitteilung implizite Eigenwerbung des Textemittenten die werbliche Themenwahl sowie die semantisch aufwertenden Strategien und sprachlichen Mitteln die Argumentation, gleichzeitig wird in der Pressemitteilung auf nachrichtentextsortentypische Weise in der Art der Themenentfaltung deskriptiv

\footnotetext{
${ }^{8}$ Neben anderen potenziellen gesellschaftlichen Teilsystemen wie der Wissenschaft oder Politik, vgl. Christoph 2008: 128ff.
} 
verfahren, da das Thema in seinen Teilkomponenten dargestellt, spezifiziert und situativ eingeordnet wird (vgl. Christoph 2008: 133). Die Beeinflussung der Textsorte Pressemitteilung macht sich auch auf der Ebene des Textsortenstils bemerkbar. Der Textgegenstand, der Textemittent, das Bezeichnete, erfährt auf der einen Seite - etwa durch den gehäuften Einsatz von Hochwertwörtern (darunter Schlüssel- und Plastikwörtern)9 ${ }^{9}$, aber auch Fremdwörtern, Fachlexik oder Adjektiven - eine semantisch Aufwertung. Zugleich sind Pressemitteilungen in der Gestaltung den Nachrichtentextsorten nachempfunden, etwa im Hinblick auf den Aufbau, das Prinzip der achronologischen Wichtigkeitsabstufung oder das Befolgen journalistischer Standards (vgl. Christoph 2008: 133f.).

Aus den textexternen Vorgaben und der erläuterten strukturellen Kopplung von zwei verschiedenen Kommunikationsbereichen ergeben sich innerhalb der Textsorte Pressemitteilung die einzelnen textinternen Merkmale: zum einen die Appellfunktion, Themenwahl nach der Strategie des Textemittenten, argumentative Themenentfaltung zur Untermauerung des Eigenwerbungscharakters sowie aufwertende Strategien und persuasive sprachliche Mittel und auf der anderen Seite die Inforationsfunktion, Themenwahl nach Nachrichtenwert, deskriptive, den Nachrichtentextsorten nachempfundene Themenentfaltung und Makrostruktur sowie journalistische Standards (vgl. Christoph 2008: 134).

Gegenüber der klassischen Pressemitteilung versteht sich die Online-Pressemitteilung ${ }^{10}$ als wichtiger Bestandteil eines erfolgreichen Content-Marketings und wesentlicher Beitrag zur Online-Sichtbarkeit des Textemittenten (vgl. Schach 2015: 2013) und ermöglicht - teils „vorbei

\footnotetext{
${ }^{9}$ Als Hochwertwörter sind nach Janich (2010: 169) alle diejenigen Ausdrücke zu bezeichnen, „die ohne die grammatische Struktur eines Komparativs oder Superlativs geeignet sind, das damit Bezeichnete (bei Substantiven) oder näher Bestimmte/Prädizierte (bei Adjektiven) aufgrund ihrer sehr positiven Inhaltsseite aufzuwerten“. Demgegenüber haben Schlüsselwörter „nicht nur aufwertende Funktion, sondern sie nehmen auch anzeigen- und produktübergreifend »eine Schlüsselstellung im Gedanken- und Sprachfeld der Werbung« ein" (Römer 1980: 132; bei Janich 2010: 169). Plastikwörter hingegen können nach Janich (2010: 171) „zugleich Schlüsselwörter sein und zeichnen sich ebenfalls durch ihre eher vage Inhaltsseite aus, sind aber mit Konnotationen einer ganz bestimmten Art verbunden“, da sie „nicht zum Wecken von Emotionen“ dienen, sondern „den Eindruck wissenschaftlicher Qualität und Fundiertheit“ verstärken, so wirken sie „verlässlich und entpuppen sich doch meist als Luftblasen“".

10 Schach (2015: 213) verwendet an dieser Stelle auch die Bezeichnungen Online-Meldung und Medienmitteilung, Schweiger/Jungnickel (2011: 400) verweisen auch auf den Einsatz von Social Media Releases im Sinne von Online-Pressemitteilungen mit zusätzlichen multimedialen Inhalten wie Fotos, Audios, Videos etc. und den Möglichkeiten zur Weitergabe in sozialen Netzwerken.
} 
an den Journalisten als bisherigen Gatekeepern"1" - durch die Distribution über PR-Portale oder Veröffentlichung auf den organisationseigenen Webseiten und Sozialen Medien direkte Adressierung der jeweiligen Ansprechgruppen (vgl. Schweiger/Jungnickel 2011: 400). Dadurch ist die Zielgruppe der Online-Meldungen daher weitaus größer als im Fall einer klassischen Pressemitteilung (vgl. Schach 2015: 2012). Den Unterschied zwischen der klassischen Massenmedienresonanz und der Onlineresonanz sehen Schweiger/Jungnickel (2011: 406) in den „offenen und heterogenen Kommunikationsstrukturen des Internets"12. Durch das (soziale) Web wird die Weitergabe von PR-Botschaften auch über die Massenmedien hinaus ermöglicht, als Multiplikatoren kommen etwa andere Organisationen oder auch die Bürger bzw. Konsumenten selbst in Frage, die die Pressemitteilungen auf ihren Websites, Facebook-Seiten oder Blogs aufgreifen (vgl. Schweiger/Jungnickel 2011: 401-405). Die Internetpräsenz des Emittenten wird im Fall von digitalen Publikationen auch durch die Aktualität und Schnelligkeit bei der Veröffentlichung ständig neuer Inhalte sowie die entsprechende Optimierung der Texte zur besseren Wirkung der Meldung bezüglich des Suchmaschinenrankings verbessert. Gegenüber der Veröffentlichungsmöglichkeit eines klassischen Pressemitteilungstextes durch eine Redaktion nur jeweils in der aktuellen Ausgabe eines Mediums, erfolgt bei der selbstveröffentlichten Online-Meldung auch eine dauerhafte Speicherung des Textes im Web (vgl. Löffler 2014: 387). Aus sprachlicher Sicht erscheint hier etwa der Einsatz von Maßnahmen zur Suchmaschinenoptimierung (Search Engine Optimization, SEO) von besonderer Relevanz, wie der Einbau von mehrfach verwendeten Schlüsselwörtern (Keywords) in der Überschrift sowie im Lead und Boilerplate, die in der Alltagssprache als normale Suchbegriffe zu dem jeweiligen Thema vorkommen oder Verlinkungen in Form von Deep Links, also einem genauen Pfad zu beschriebenem Dokument oder Datei (vgl. Zehrt 2014: 197-198 und Schach 2015: 212-213; siehe auch Solis/Breakenridge 2010).

\footnotetext{
${ }^{11}$ Schweiger/Jungnickel verweisen in diesem Zusammenhang auf White und Raman, die bereits 1999 das Internet als „first public relations mass medium in that it allows managed communication to flow directly between organizations and mass audiences without the gatekeeping function of other mass media" (White/Raman 1999: 406; bei Schweiger/Jungnickel 2011: 400, Hervorhebungen J.M.)

${ }^{12}$ Dem beschränkten Rahmen des vorliegenden Beitrags halber wird auf das bislang relativ spärlich untersuchte Phänomen der Massenmedien- und Onlineresonanz von (Online-)Pressemitteilung, wie etwa die Rolle von Presseportalen als einer Art „Relaisstationen für Organisationsbotschaften“, nicht weiter eingegangen, siehe hierzu u. a. Schweiger/Jungnickel 2011.
} 
Die Politolinguistik befasst sich nach Girnth/Hofmann (2016) mit dem Politikbegriff im möglichst weitesten Sinn, damit werden unter Politik „nicht nur die von politischen Funktionsträgern produzierten Äußerungen und Texte (enger Politikbegriff) und die öffentliche Kommunikation in den Medien berücksichtigt (weiter Politikbegriff), sondern das Reden aller Mitglieder einer Sprachgemeinschaft über politisch relevante Sachverhalte“ (vgl. Girnth/Hofmann 2016: 8; vgl. auch Schröter/Carius 2009: 10ff.). Geht man aus politolinguistischer Sicht von dem Politikbegriff im weitesten Sinn aus, so versteht sich auch die innerhalb politischer Kommunikation eingesetzte klassische Pressemitteilung bzw. Online-Pressemitteilung als politische Textsorte. Roth sieht dabei in der Pressemitteilung ein ständig im politischen Sprachgebrauch vorhandenes Kommunikationsinstrument, denn während im Laufe der Legislaturperiode sich Politik „durch Presseerklärungen, Interviews, den Parlamentsbetrieb, durch Nachrichten- und Magazinsendungen der elektronischen Medien, durch Zeitungsnachrichten und Kommentare [,verkauft'], kommen „fast ausschließlich während des Wahlkampfes [...] Plakate, Inserate, Prospekte, Kleinwerbemittel und Werbespots hinzu"(Roth 1987: 298). Bei Betrachtung einiger in der einschlägigen Literatur vorgenommener Klassifikationsversuche von Textsorten im politischen Sprachgebrauch werden Pressemitteilungen im engen Sinn jedoch als nicht-genuine politische Textsorten betrachtet. Auch wenn sie, so Tillmann (1989: 61), ebenso „von politisch Handelnden zur Realisierung ihrer kommunikativen Ziele verwendet werden können“, werden sie nicht in die Taxonomie von Textsorten politischer Sprache integriert, zumal sie auch in anderen Praxisbereichen der Kommunikation Anwendung finden können. Genuine politische Textsorten werden dagegen „ausschließlich von politisch Handelnden zum Zweck der parteilich-politischen Kommunikation realisiert" (Tillmann 1989: 61). Vor dem Hintergrund einer solchen methodologischen Ausgrenzung wird in diesem Fall - neben ,in zwischenstaatlicher Kommunikation relevanten politischen“ sowie "normativen juristischen Textsorten“ - die Pressemitteilung in der Kategorie der publizistischen Textsorten (unter der Bezeichnung Komuniquê) und in der Subkategorie der Informationstexte neben Meinungstexten und expressiven Texten aufgeführt (vgl. Tillmann 1989: 56-58). Die publizistischen Textsorten werden somit zwar als nichtoriginäre politische Textsorten klassifiziert, ihnen wird aber im „Gesamtzusammenhang parteilich-politischen Handelns eine nicht unbedeutende Rolle“ zugeschrieben (vgl. Tillmann 1989: 57). Bei seiner Klassifikation von Textsorten im Bereich politischer Institutionen geht Klein (vgl. 2000: 734) von der Tatsache aus, dass die Funktionen und Rollen, welche das System 
moderner parlamentarischer Demokratien zuweist, sich zu einem hohen Grand in der „ausschließlichen oder dominanten Zuständigkeit als Emittent" bestimmter Textsorten manifestieren. Als „Träger solcher Systemfunktionen" und damit Emittenten von Klassen politisch relevanter Textsorten verstehen sich Parlamente und „parlamentähnliche“ Versammlungen, Regierungen, Parteien, politische Funktionsträger als Personen und personale Repräsentanten, externe Emittenten von politisch relevanten Textsorten wie Verfassungsgerichte, Verbände, Experten und Presse (Klein 2000: 734). Als emittentenspezifisch unterscheidet Klein (2000: 736-754) somit von Volksvertretungen, Regierungen, Parteien/Fraktionen, Politikern als personalen Repräsentanten emittierte Textsorten sowie politikadressierte Textsorten externer Emittenten und emittentenunspezifische Textsorten, zu denen als mediale Textsorten neben etwa Leserbrief, Interview, Gastkommentar auch Pressemitteilungen hinzugezählt werden. Der Großteil schriftlicher Texte, die, so Klein (2000: 754), ,politische Institutionen und ihr Personal emittieren, gehören TS an, die nicht politikspezifisch sind und die in allen Institutionen und Berufsgruppen mit Öffentlichkeitsbezug verwendet werden“. Dabei ließe sich allerdings keine durchgängige spezifische Ausprägung der Textsorte erkennen, welche die Klassifizierung als einer politischen Textsorte legitimieren würde, auch wenn der „einzelne Text thematisch und strategisch durch die politischen Diskursverflechtungen und Intentionen des jeweiligen Emittenten geprägt sein mag“" (Klein 2000: 754).

\section{Zur Pilotstudie und Untersuchungskorpus}

Die im vorliegenden Beitrag beschriebene durchzuführende Pilotstudie basiert auf einem Untersuchungskorpus von insgesamt 60 Online-Pressemitteilungen ${ }^{13}$, die im Zeitraum vom 1. bis 28 . Februar 2018 auf der offiziellen Webseite der Partei Alternative für Deutschland (AfD) in der Sparte Pressemitteilungen veröffentlicht wurden ${ }^{14}$. In Anlehnung an den vorhergehenden textlinguistisch orientierten Systematisierungsversuch der Textsorte Online-Pressemitteilung wird auf der Vorlage von dem zur Beschreibung von politischen Textsorten weitverbreiteten Kategorieinventar von Klein

\footnotetext{
${ }^{13}$ Laufend nummeriert von T01 bis T60, vgl. Anhang zu diesem Beitrag.

${ }^{14}$ Das Untersuchungskorpus umfasst alle zum Zeitpunkt der Datenerhebung im Pressearchiv auf der Webseite der Alternative für Deutschland (AfD) im Zeitraum Februar 2018 aufgeführten Online-Pressemitteilung. Quelle: www.afd.de/2018/02/, Breadcrumb: www.afd.de $\rightarrow$ Presse $\rightarrow$ Pressemitteilungen $\rightarrow$ Pressearchiv $\rightarrow$ Februar 2018. Als Zeitpunkt der Datenerhebung gilt der 14. März 2018.
} 
(2000)15 der Versuch unternommen, den Einsatz der Textsorte Online-Pressemitteilung im politischen Sprachgebrauch unter pragmatischen, semantischen, lexikalischen, grammatischen und rhetorischen Kriterien zu ermitteln und zu beschreiben. Im Folgenden werden im Einzelnen die quantitativen textexternen und -internen Parameter zu dem im Untersuchungskorpus exzerpierten Textmaterial dargestellt, dem schließt sich eine exemplarische Textanalyse eines gewählten Online-Pressemitteilungstextes an.
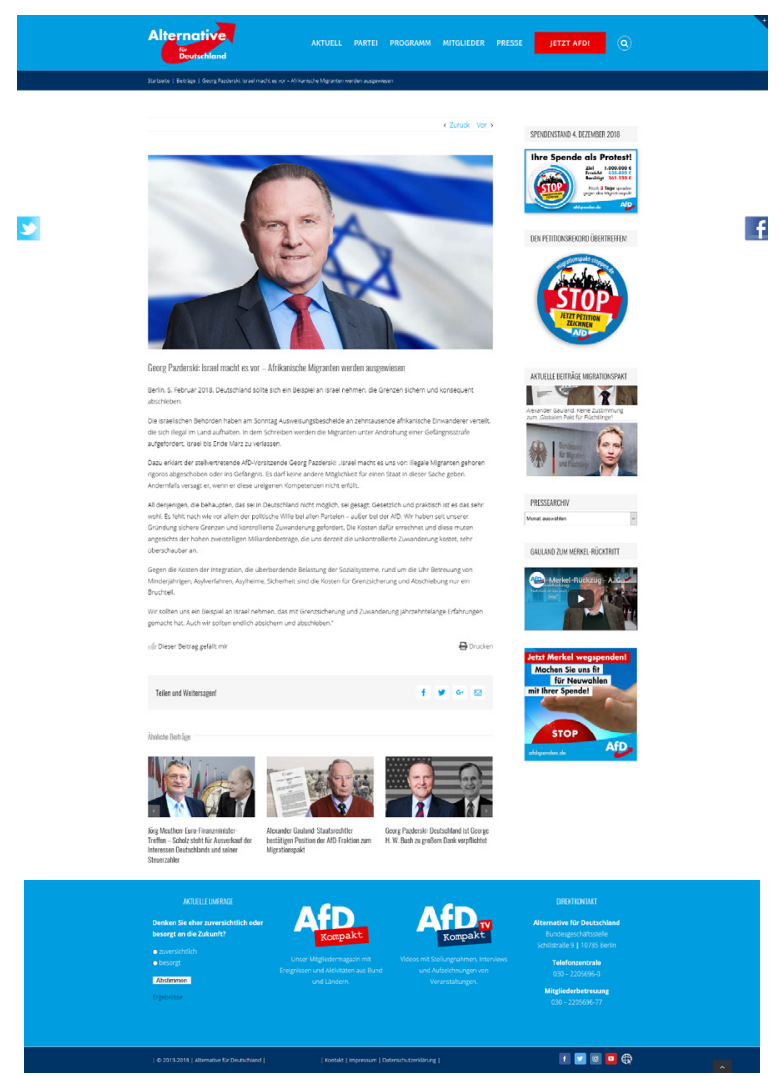

Abb. 2. Exemplarische Online-Pressemitteilung vom 5. Februar 2018 u. d. T. Georg Pazderski: Israel macht es vor - Afrikanische Migranten werden ausgewiesen (T16, 1678 Zeichen, 6 Absätze) im Website-Layout

Quelle: www.afd.de/georg-pazderski-israel-macht-es-vor-afrikanische-migranten-werden-ausgewiesen/ (05.12.2019).

15 Vgl. hierzu u. a. Pappert (2017) zur TS Wahlplakat, siehe auch Fix (2014) zur TS Leserbrief oder Makowski (2013) zur TS Abgeordnetenrede im Europäischen Parlament. 


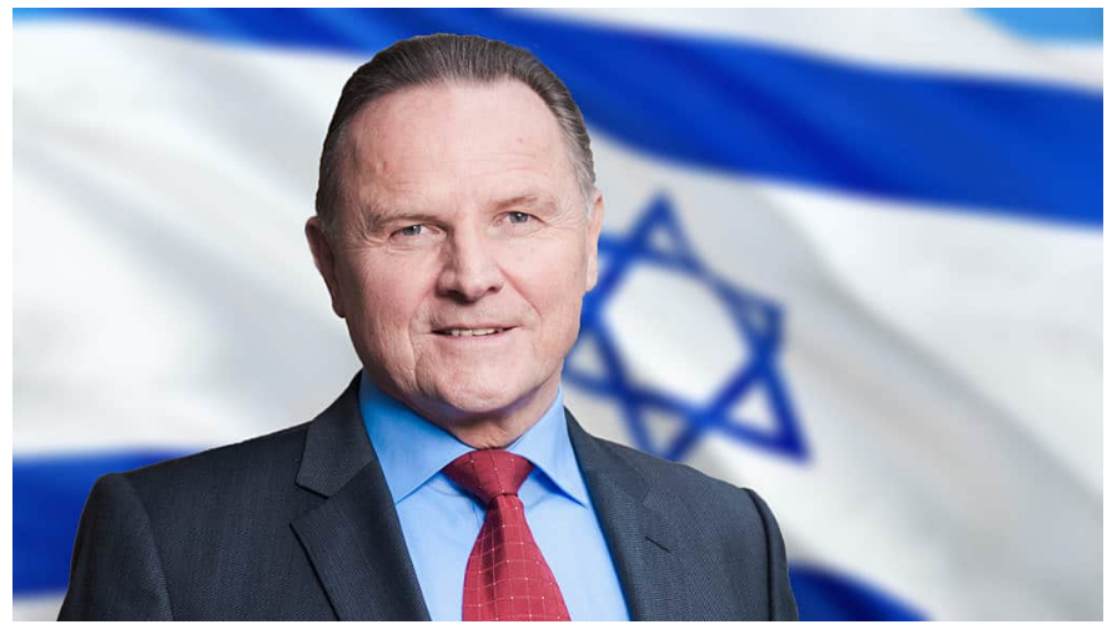

Georg Pazderski: Israel macht es vor - Afrikanische Migranten werden ausgewiesen

Berlin, 5. Februar 2018. Deutschland sollte sich ein Beispiel an Israel nehmen, die Grenzen sichern und konsequent abschieben.

Die israelischen Behörden haben am Sonntag Ausweisungsbescheide an zehntausende afrikanische Einwanderer verteilt, die sich illegal im Land aufhalten. In dem Schreiben werden die Migranten unter Androhung einer Gefängnisstrafe aufgefordert, Israel bis Ende März zu verlassen.

Dazu erklärt der stellvertretende AfD-Vorsitzende Georg Pazderski: „Israel macht es uns vor: Illegale Migranten gehören rigoros abgeschoben oder ins Gefängnis. Es darf keine andere Möglichkeit für einen Staat in dieser Sache geben. Andernfalls versagt er, wenn er diese ureigenen Kompetenzen nicht erfüllt.

All denjenigen, die behaupten, das sei in Deutschland nicht möglich, sei gesagt: Gesetzlich und praktisch ist es das sehr wohl. Es fehlt nach wie vor allein der politische Wille bei allen Parteien - außer bei der AfD. Wir haben seit unserer Gründung sichere Grenzen und kontrollierte Zuwanderung gefordert. Die Kosten dafür errechnet und diese muten angesichts der hohen zweistelligen Milliardenbeträge, die uns derzeit die unkontrollierte Zuwanderung kostet, sehr überschaubar an.

Gegen die Kosten der Integration, die überbordende Belastung der Sozialsysteme, rund um die Uhr Betreuung von Minderjährigen, Asylverfahren, Asylheime, Sicherheit sind die Kosten für Grenzsicherung und Abschiebung nur ein Bruchteil.

Wir sollten uns ein Beispiel an Israel nehmen, das mit Grenzsicherung und Zuwanderung jahrzehntelange Erfahrungen gemacht hat. Auch wir sollten endlich absichern und abschieben."

Abb. 3. Exemplarische Online-Pressemitteilung vom 5. Februar 2018 u. d. T. Georg Pazderski: Israel macht es vor - Afrikanische Migranten werden ausgewiesen (T16, 1678 Zeichen, 6 Absätze)

Quelle: www.afd.de/georg-pazderski-israel-macht-es-vor-afrikanische-migranten-werden-ausgewiesen/ (05.12.2019). 
a) Angaben zum Untersuchungskorpus

- Textsorte: Online-Pressemitteilung,

- Emittent: Partei (Alternative für Deutschland) ${ }^{16}$,

- Adressat: breit gefasste Öffentlichkeit, prinzipiell als nicht auf Wahlkämpfe beschränkte Textsorte, ggf. andere politische Funktionsträger und/oder Bürger als Wähler in Wahlkampzeiten ${ }^{17}$,

- Textart18: prototypisch schriftlich konzipierte und realisierte Textsorte,

- Umfang: insgesamt 60 auf der parteieigenen Webseite veröffentlichte Online-Pressemitteilungen (T01 - T60),

- Zeitlicher Rahmen: 1. bis 28. Februar 2018,

- Zeitpunkt der Datenerhebung: 14. März 2018.

b) Layout und Aufbau

- Logo: nicht unmittelbarer Bestandteil der Textstruktur, vorhanden als Element der Webseite, links oben,

- Textsortenkennzeichnung: nicht explizit gekennzeichnet, unmittelbarer Verweis durch Lokalisierung des Textes in der Unterseite Presse und der Subkategorie Pressemitteilungen,

- Sperrfrist: nicht explizit gekennzeichnet, nicht mehr aktuelle Pressemitteilungen werden aus dem Online-Pressearchiv herausgenommen. Für den Zeitraum Februar 2018 befanden sich im Archiv um

${ }^{16}$ Explizit markiert durch Angaben im Impressum: Angaben gemäß $§ 5$ TMG / Alternative für Deutschland (AfD) / vertreten durch den Bundesvorstand, / dieser gemäß § 14 (3) Bundessatzung vertreten durch die Bundessprecher: Prof. Dr. Förg / Meuthen und Dr. Alexander Gauland, Verantwortlich für den Inhalt nach $\$ 55 \mathrm{Abs}$. 2 RStV / Alternative für Deutschland (AfD) / vertreten durch den Bundesvorstand, / dieser gemäß § 14 (3) Bundessatzung vertreten durch die Bundessprecher: Prof. Dr. Förg / Meuthen und Dr. Alexander Gauland, www.afd. de/impressum/ (15.12.2018).

17 Dieckmann verweist im Hinblick auf die oft thematisierte Mehrfachgerichtetheit politischer Kommunikation der politischen Funktionsträger auf die Trialogizität politischen Sprachgebrauchs (vgl. Dieckmann 1981: 265ff.) Die Mehrfachadressierung politischer Reden kommt etwa dadurch zum Tragen, dass sich der jeweilige Redner zugleich an die versammelte Zuhörerschaft oder Plenum sowie die über die Medien präsente breite Öffentlichkeit wendet (vgl. Burkhardt 2003: 277). Parlamentarische Plenarkommunikation kann beispielsweise auf eine recht vielschichtige Art mehrfachadressiert sein, wenn sich der Emittent zum einen an die physisch anwesenden Zuhörer („innere Öffentlichkeit") über an die über die Medien teilnehmende Öffentlichkeit der Bürger (,äußere Öffentlichkeit") sowie zum anderen etwa an die politisch Verantwortlichen anderer Staaten („externe Öffentlichkeit“) wendet (vgl. Burkhardt 2003: 281, siehe hierzu auch Makowski 2013: 125f.).

${ }^{18}$ Das Kriterium Textart (vgl. Klein 2000: 735) geht mehr oder minder mit der Kommunikationsform nach Brinker (vgl. 2005: 146-150) einher, vgl. hierzu auch Koch/Oesterreicher 2008. 
Zeitpunkt der Datenerhebung insgesamt 60 Pressemitteilungen, zum aktuellen Zeitpunkt (25.04.2019) enthält das Archiv 19 Texte,

- Schlagzeile: gebildet nach dem Muster: AfD-Prominenter/-Funktionär + Hauptthema. Dachzeile und Unterzeile sowie Zwischenüberschriften nicht vorhanden, Hervorhebung durch Schriftzeichengröße,

- Vorspann (Lead): immer vorhanden, erster Absatz, jeweils eingeleitet durch Spitzmarke mit Orts- und Zeitangabe, keine gesonderte Hervorhebung,

- eigentlicher Text: immer vorhanden, jeweils im Anschluss an den Vorspann, im Umfang von einem bis neun Absätzen von unterschiedlicher Länge,

- Textumfang (inklusive Leerzeichen und Titel): der Umfang der Texte im Untersuchungskorpus beträgt von 733 bis 6826 Zeichen bei einer durchschnittlichen Textlänge von 1759 Zeichen. Es konnten keine Texte im Umfang von bis 200 Zeichen festgestellt werden, es wurden zwölf Texte im Umfang von 400 bis 1200 Zeichen, 31 Texte im Umfang von 1200 bis 2000 Zeichen, zwölf Texte im Umfang von 2000 bis 2800 Zeichen und fünf Texte im Umfang von über 2800 Zeichen verzeichnet,

- Gliederung: die Texte werden in Absätze von unterschiedlicherer Zahl und Umfang mit einleitendem Vorspann (Lead) gegliedert. Durchschnittliche Zahl der Absätze beträgt 5,15.

c) Zusätzliche Informationen

- Längenangabe: nicht vorhanden,

- Abbinder (Boilerplate): kein unmittelbarer Bestandteil der Textstruktur, vorhanden als Element der Webseite, unter den einzelnen Unterseiten wie Partei oder Impressum abrufbar,

- Kontaktdaten, Ansprechpartner: kein unmittelbarer Bestandteil der Textstruktur, vorhanden als Element der Webseite, unter den einzelnen Unterseiten wie Partei, Impressum, Kontakt abrufbar,

- Video-, Bildmaterial: Bild/Graphik als Bestandteil der Textstruktur, einleitend, unmittelbar vor der Schlagzeile (58 von 60 Textbeispielen). Als Abweichungen von diesem Muster gelten die Textbeispiele T10 - Bild/Graphik erscheint unmittelbar nach der Schlagzeile - und T43, wo unmittelbar nach der Schlagzeile statt einem statischen graphischen Content ein Video platziert wird,

- Links, Verweise auf weitere Informationen, Downloadmöglichkeiten etc.: zum Zeitpunkt der Datenerhebung direkt unterhalb des Textes durch den Button Drucken und die Leiste Teilen und 
Weitersagen mit Verlinkungen zu Facebook, Twitter, Instagram, Reddit, Tumbrl, Google Plus, Pinterest, VKonakte, Mail. Zum aktuellen Zeitpunkt (25.04.2019): direkt unterhalb des Textes durch das Button Gefällt mir sowie die angeschlossene Mitteilung 1 Personen gefällt dieser Beitrag. Und Ihnen?, das Button Drucken und die Leiste Teilen und Weitersagen mit Verlinkungen zu Facebook, Twitter, Google Plus, Mail.

\section{Exemplarische Textanalyse}

Nach der vorhergehend genannten Vorlage des Kategorieninventars zur Beschreibung politischer Textsorten nach Klein (2000) erfolgt nachstehend die exemplarische Analyse des Textbeispiels förg Meuthen: Rund 1.000 Besucher beim Politischen Aschermittwoch der AfD vom 16. Februar 2018 (T43, siehe Anhang). Innerhalb der jeweiligen Kategorien wird insbesondere auf die für die vorliegende Kommunikationssituation relevanten Kriterien eingegangen.

a) Pragmatische Kategorien:

- Emittent: Alternative für Deutschland als Partei/Fraktion,

- Adressat: breit gefasste Öffentlichkeit sowie Bürger als Wähler aufgrund der Wahlkampzeit und thematischer Bezüge zum Wahlkampf, indirekt auch auf- bzw. abwertend deutsche und österreichische politische Funktionsträger,

- Textart: prototypisch schriftlich konzipierte und realisierte Textsorte, abwechselnd Merkmale geschriebener und gesprochener Sprache (vgl. nachstehend Erörterungen zur Syntax),

- Grundfunktion: unmittelbar aus der Information über die Veranstaltung des politischen Aschermittwochs als Hauptthema hervorgehende Diskreditierung politischer Wahlgegner sowie Profilierung eigener Fraktion und Funktionäre,

- Texthandlungsmuster:

- Initiatoren: Überschrift, Spitzmarke mit Orts- und Zeitangabe,

- Texteinleitung (Absatz 1, Vorspann): DISKREDITIEREN (SPD, CSU), INFORMIEREN,

- Textkern: Absatz 2 - INFORMIEREN, Absatz 3 - INFORMIEREN, PROFILIEREN, Absatz 4 - INFORMIEREN, DISKREDITIEREN (die Linke), Absatz 5 - DISKREDITIEREN (SPD), Absatz 6 - DISKREDITIEREN (CDU, kleinere Parteien), Absatz 7 - INFORMIEREN, PROFILIEREN, Absatz 8 - KRITISIEREN, 
DISKREDITIEREN (CSU), Absatz 9 - DISKREDITIEREN (CSU), INFORMIEREN, PROFILIEREN,

- Textausleitung: Absatz 10 - DISKREDITIEREN (CSU), PROFILIEREN, ZUSAMMENFASSENDES BEWERTEN,

- Terminatoren: keine gesonderte Markierung,

- Geltungsmodus: offiziell und öffentlich,

- TS-Intertextualität: als Nach-Textsorte direkter Zusammenhang zu den bei der im Rahmen der Veranstaltung des politischen Aschermittwochs vom 14. Februar 2018 gehaltenen Wahlkampf-/Parteitagsreden von AfD-Parteifunktionären.

b) Semantische Kategorien:

- Thema: als Hauptthemen (1) Großveranstaltung des Politischen Aschermittwochs der AfD (Überschrift, Vorspann und drei weitere Absätze) und (2) Diskreditierung/Kritik an politischen Gegnern (überwiegend SPD, CDU und CSU und deren prominente Funktionäre) im Kontext des Wahlerfolgs bei den Bundestagswahlen und des bayerischen Landtagswahlkampfs (Vorspann und sechs weitere Absätze) sowie (3) Migrationspolitik, (4) Energiepolitik und (5) Kooperation mit der FPÓ als Subthemen zu (1) und (2),

- Lexik: bei praktisch nicht feststellbaren Fahnenwörtern und einer geringen Anzahl von auf den Emittenten und dessen politischen Partner gerichteten Hochwertwörtern (Volksfeststimmung, Gemeinsamkeiten, Wende, Zukunft) eine überwiegende Zahl von Stigma-, Unwert- und Scheltwörtern ${ }^{19}$ gegenüber politischer Konkurrenz (der gescheiterte Hoffnungsträger, Groko-Gruselkoalition, rote FreakShow, Maas-Männchen, Noch-Kanzlerin, Noch-CDU-Vorsitzende, eine sadistische Domina, die personifizierte Schlaftablette, politische Nischen-Anbieter, selbstverliebte Christian-Lindner-Partei, Allah-CSU, Flüchtling in Merkels Regierung, kleine regionale Voralpen-Partei, Altparteien, Rassismus gegen die eigene Bevölkerung, Dampfplauderei, Islamierung Bayerns, Flächenfraß), innerhalb politischen Instituti-

\footnotetext{
19 Als Subkategorie des politischen Schlagworts unterscheidet Burkhardt (2003) die parteisprachlichen Fahnenwörter zur Kennzeichnung eigener politischer und gesellschaftlicher Programme sowie Stigmawörter als Ausdruck von Negativ-Bezeichnungen gegnerischer Parteien, deren Mitgliedern, Zielen und Werten, die überparteilichen zeitlos über der aktuellen politischen Diskussion stehenden Hochwertwörter und die parteiübergreifenden und negativierenden Unwertwörter sowie Scheltwörter als aus der Perspektive einer Partei geäußerte beschränkte Abwertung mit dem Vorwurf moralischer Verfehlung (vgl. Burkhardt 2003: 352-359).
} 
onsvokabulars ${ }^{20}$ zahlreich vorkommende Bezeichnungen für staatliche Organisationen (Deutsche[r] Bundestag, Landtag, Regierung, Parteien), staatliche und politische Rollen (Landeschef, Generalsekretär, Bezirksvorsitzende, Parteichef, Abgeordneter im Europaparlament, Bundestagsabgeordnete) und politik-spezifische Bezeichnungen für politische Handlungen, Prozesse und Zustände (Landtagswahlkampf, Parteitage, Bundestagswahl) sowie gesondert Fachausdrücke aus anderen Bereichen (Krankenhausplätze, Kostengründe, Asylbewerberheime, Energiepolitik, Abholzung, Windräder) und Regionalismen (Brezeln, Weißwürste, Dirndl, Trachten, Lederhosen), gehäuft Formulierungen aus der Alltags- und Umgangssprache (im Landtag sitzen, Politik machen, die CSU [habe] die Lederhosen gestrichen voll, Die Altparteien haben den Klimawandel komplett verpennt).

c) Grammatische Kategorien:

- Syntax: prototypisch schriftlich konzipierte und realisierte Textsorte, hier: als Nach-Textsorte zur mündlich realisierten Rede (Wahlkampf-/Parteitagrede), wörtliche Zitate aus gesprochener Sprache. Prinzipiell schriftlich konzipierte Textsorte, da allerdings der Text überwiegend auf wortwörtliche oder paraphrasierte Zitierungen aus mündlich konzipierten Textsorten (Wahlkampf-/Parteitagreden während der beschriebenen Großveranstaltung), sind im Text abwechselnd Merkmale geschriebener und gesprochener Sprache $\mathrm{zu}$ isolieren. Während der Vorspann und die weiteren vier Absätze sich vielmehr an der geschriebenen Sprache orientieren, ist in den weiteren sechs Absätzen häufiger Wechsel zwischen geschriebener und gesprochener Sprache zu verzeichnen,

- Personenbezug durch Personalformen: das inklusive wir nur in zitierten Redeabschnitten gruppenintegrativ parteibezogen (In Bayern gehört der Islam nicht zu Deutschland und nur mit uns bleibt die Kirche im Dorf, Denn wir ziehen auf jeden Fall in den Landtag ein, das ist so sicher wie das Amen in der Kirche) und als Ausdruck von Partnerschaft, Kooperation und Gemeinsamkeit im Hinblick auf die AfD-FPÖ-Relation (Die Zukunft gehört uns, wenn wir uns selbst treu bleiben), sowie integrierend wähler-/bürgergerichtet (Statt einer Willkommenskultur für illegale Einwanderer brauchen wir eine Willkommensstruktur für Kinder).

${ }^{20} \mathrm{Zu}$ fachsprachlichen Zügen des politischen Institutionsvokabulars in Verbindung mit der Politikersprache siehe Klein 1989: 6. 
Rhetorische Kategorien:

- Bauform:

- Schlagzeile gebildet nach dem Muster AfD-Prominenter/-Funktionär + Hauptthema ohne Dach- und Unterzeile,

- Video u. d. T. Förg Meuthen beim Politischen Aschermittwoch in Bayern mit Verlinkung zum offiziellen AfD-Youtube-Kanal,

- Vorspann (Lead) vorhanden und eingeleitet durch Spitzmarke mit Orts- und Zeitangabe ohne gesonderter Hervorhebung,

- eigentlicher Text im Umfang von 9 Absätzen von unterschiedlicher Länge, Textumfang (inklusive Leerzeichen und Titel) beträgt 6826 Zeichen,

- Themenentfaltung: dominierende argumentative thematische Entfaltung mit emotiv-bewertenden Formen,

- Rhetorische Figuren/Tropen: zahlreiche rhetorische Figuren, metaphorische und metonymische Ausdrücke, Anspielungen, Wortspiele, Spiel mit Eigennamen und idiomatische Formulierungen, die teils einmalig und teils um ein sich wiederholendes Motiv gebildet werden. Ins Gewicht fallen zum einen auf Imagepflege ausgerichtete Eingriffe, wie etwa teils metaphorische mehrfach wiederholte positive Rückkopplung des Publikums (bekam...stürmischen Applaus, unter starkem Beifall, Tosenden Applaus und Bravo-Rufe gab es..., Zu den lang anhaltenden lautstarken „,Merkel muss weg"-Sprechchören..., unter Beifall, unter Applaus, unter lautem Beifall) bei gleichzeitiger Diskreditierung politischer Konkurrenz in demselben Bereich (applaudiere man ihr [Angela Merkel] bei Parteitagen artig wie die „Duracell-Klatschhasen "), Formulierungen rund um die Metaphorik das politischen Wahlkamps als Krieg/Sport (nach einer ununterbrochenen Siegesserie, das beste westdeutsche Ergebnis, sie [die AfD]...machte Deggendorf zur AfD-Hochburg, Denn wir ziehen auf jeden Fall in den Landtag ein), in denen die gegnerische Seite im Sinne der Metaphorik des Untergangs/Zerfalls dargestellt wird (in der Wählergunst abstürzende SPD, Niedergang dieser einst großen konservativen Partei, Und wenn die SPD sich selbst zerlegt, in der Wählergunst sinkende CSU), auf der anderen Seite in bedeutender Zahl auf Diskreditierung der politischen Gegner abzielende Eingriffe wie Anspielungen auf die Migrationspolitik der CDU/CSU (bei der CSU symbolisiert der Buchstabe "C" inzwischen die islamische Halbmond-Sichel anstelle des Christentums, Sie möchte weiterhin lieber Miniröcke statt Burka sehen, In Bayern gehört der Islam nicht zu Deutschland und 
nur mit uns bleibt die Kirche im Dorf), Nachweis der Inkonsequenz und Handelsunfähigkeit rund um die Dampf-/Heißluft-Metaphorik (Heißluft-Horst Seehofer, Mit der Dampfplauderei der CSU ist bald Schluss), Entlarvung und Diskriminierung gegnerischer politischer Funktionsträger (Angela Merkel als sadistische Domina oder personifizierte Schlaftablette, Horst Seehofer als Heißluft-Horst oder Flüchtling in Merkels Regierung, Heiko Maas als Maas-Männchen), ferner mehrere intensivierende idiomatisierte Ausdrücke in beiden Funktionen (Neben SPD und Union bekamen auch die kleineren Parteien ihr Fett weg, Asylbewerberheime [schossen] wie Pilze aus dem Boden, die [CSU] werden ihr blaues Wunder erleben, die CSU [habe] die Lederhosen gestrichen voll, Denn wir ziehen auf jeden Fall in den Landtag ein, das ist so sicher wie das Amen in der Kirche, Die Altparteien haben den Klimawandel komplett verpennt, nicht den am Nordpol, sondern den gesellschaftlichen und politischen Klimawandel in Deutschland und in Bayern."

\section{Fazit und Ausblick}

Im Hinblick auf die skizzierte Fragestellung des vorliegenden Beitrags als dem ersten Schritt zu einer Pilotstudie zum Einsatz von Online-Pressemitteilungen im politischen Marketing konnte im Rahmen der durchgeführten exemplarischen Analyse eines der offiziellen Webseite der Alternative für Deutschland entnommenen Textbeispiels die Annahme bestätigt werden, das zumindest im gegebenen Fall die Textsorte Pressemitteilung im politischen Sprachgebrauch entgegen ihrer prototypischen Funktion zum Zweck der Diskreditierung politischer Gegner missbraucht werden kann.

Unmittelbar aus der Information über die Wahlveranstaltung als angekündigtes Hauptthema geht die Profilierung der eigenen Fraktion und deren Funktionäre sowie die dominierende Diskreditierung politischer Wahlgegner hervor. Dies belegen die innerhalb der Kategorie Texthandlungsmuster isolierten dominierenden Sprachhandlungen (DISKREDITIEREN, PROFILIEREN), der trotz des im Titel und Vorspann angekündigten informativen Charakters im ersten Absatz sowie sechs weiteren Absätzen dominierenden Diskreditierung und Kritik an politischer Konkurrenz als eigentlichem Themenschwerpunkt, ferner auch die im lexikalischen wie rhetorischen Bereich isolierten unzähligen auf Herabsetzung und Abwertung politischer Gegner abzielenden Eingriffe. Angesichts der prototypisch überwiegend informativen und imagepfle- 
genden Hauptfunktion der Textsorte Pressemitteilung versteht sich der in diesem Fall zweifelsohne zweckmäßige Einsatz diskreditierender und semantisch abwertender Strategien als eindeutig manipulativ.

Bei der Betrachtung des Phänomens von Hassrede in Online-Pressemitteilungen als einer Strategie der Diskreditierung politischer Gegner bleibt es selbstverständlich weiteren Untersuchungen vorbehalten, inwiefern sich die im vorliegenden Beitrag belegte Tendenz (1) als eine konsequent durch den Textemittenten Alternative für Deutschland in der gesamten Sparte Pressemitteilungen eingesetzte Strategie handelt und (2) ob die festgestellte Tendenz einzig und allein bei dem vorliegenden Emittenten vorkommt, oder aber auch generell im politischen Sprachgebrauch durch politische Parteien zum Einsatz kommt, (3) ferner auch ob und inwieweit sich der Einsatz der Strategie der Diskreditierung innerhalb der Textsorte nach äußeren Umständen richtet, wie etwa relevanten politischen Geschehnissen, der Wahlkampfzeit etc.

\section{Literaturverzeichnis}

Bachmann, Cornelia (1997): Public Relations und Ghostwriting für Medien. Eine linguistische Analyse der journalistischen Leistung bei der Adaptation von Pressemitteilungen. Bern.

Baechler, Coline et al. (Hg.) (2016): Medienlinguistik 3.0 - Formen und Wirkung von Texten im Zeitalter des Social Web. Berlin.

Bischl, Katrin (2015): Die professionelle Pressemitteilung. Ein Leitfaden für Unternehmen, Institutionen, Verbände und Vereine. 2., aktualisierte und erweiterte Auflage. Wiesbaden.

Bonacchi, Silvia (2017): Verbale Aggression. Multidisziplinäre Zugänge zur verletzenden Macht der Sprache. Berlin/Boston.

Bruhn, Manfred (2005): Kommunikationspolitik. Systematischer Einsatz der Kommunikation für Unternehmen. 3., überarbeitete Auflage. München.

Burkhardt, Armin (2003): Das Parlament und seine Sprache. Studien zu Theorie und Geschichte parlamentarischer Kommunikation. Tübingen.

Christoph, Cathrin (2008): Die Pressemitteilung als Textsorte der strukturellen Kopplung von Wirtschaft und Journalismus. In: Gansel, Christina (Hg.): Textsorten und Systemtheorie. Göttingen, S. 119-138.

Christoph, Cathrin (2009): Textsorte Pressemitteilung. Zwischen Wirtschaft und Journalismus. Konstanz.

Deg, Robert (2017): Basiswissen Public Relations. Professionelle Presse- und Öffentlichkeitsarbeit. 6., überarbeitete und erweiterte Auflage. Wiesbaden.

Dieckmann, Walther (1975): Sprache in der Politik. Einführung in die Pragmatik und Semantik der politischen Sprache. Zweite Auflage. Heidelberg. 
Dieckmann, Walther (1981): Politische Sprache. Politische Kommunikation. Vorträge. Aufsätze. Entwürfe. Heidelberg.

Falkenberg, Viola (2006): Pressemitteilungen schreiben. Zielführend mit der Presse kommunizieren. Mich Checklisten und Übungen zur Kontrolle. 4. Auflage. Frankfurt am Main.

Finkbeiner, Rita/Meibauer, Jörg/Wiese, Heike (Hg.) (2016): Pejoration. Amsterdam/Philadelphia.

Fix, Ulla (2014): Sprache, Sprachgebrauch und Diskurse in der DDR. Ausgewählte Aufsätze. Berlin.

Franck, Norbert (2017): Praxiswissen Presse- und Öffentlichkeitsarbeit. Ein Leitfaden für Verbände, Vereine und Institutionen. 3., aktualisierte und erweiterte Auflage. Wiesbaden.

Gabler, Verlag/Springer, Fachmedien Wiesbaden GmbH (2010) (=Gabler 2010): Gabler Kompakt-Lexikon Wirtschaft: 4500 Begriffe nachschlagen, verstehen, anwenden. 10., vollständig überarbeitete und erweiterte Auflage. Wiesbaden.

Gerd, Antos (2017): Fake News. Warum wir auf sie reinfallen. Oder: »Ich mache euch die Welt, so wie sie mir gefällt«. In: Der Sprachdienst 1, S. 1-20.

Girnth, Heiko/Hofmann, Andy Alexander (2016): Politolinguistik. Heidelberg.

Greule, Albrecht (2017): Vom Satz zum Text. Tübingen.

Hagl, Carolin (2011): „Ganz schön stressig!“ - Persuasive Strategien in Politischen Kinder-Online-Sachtexten. In: Makowski, Jacek (Hg.) (2011): How not to Do Things with Words. Beiträge zur Sprache in Politik, Recht und Werbung. Łódź, S. 69-83.

Holly, Werner (2003): Die Ordnung des Skandals. Zur diskursanalytischen Beschreibung eines ,Frame' am Beispiel der CDU-Spendenaffäre. In: Burkhardt, Armin: Politik, Sprache und Glaubwürdigkeit. Linguistik des politischen Skandals. Wiesbaden, S. 47-68.

Janich, Nina (Hg.) (2008): Textlinguistik. 15 Einführungen. Tübingen.

Janich, Nina (2010): Werbesprache. Ein Arbeitsbuch. 5., vollständig überarbeitete und erweiterte Auflage. Tübingen.

Kämper, Heidrun/Wengeler, Martin (Hg.) (2017): Protest - Parteischelte - Politikverdrossenheit: Politikkritik in der Demokratie. Bremen.

Kießling, Daniel/Zolleis, Udo (2005): Politische Werbung in modernen Wahlkampagnen. Zu den Kriterien professioneller Werbestrategien. In: Vogel, Bernhard (Hg.): Die Politische Meinung 1, Nr. 422. Osnabrück, S. 47-55.

Klein, Josef (1996): Dialogblockaden. Dysfunktionale Wirkungen von Sprachstrategien auf dem Markt der politischen Kommunikation. In: Klein, Josef/Diekmannshenke, Hajo (Hg.), Sprachstrategien und Dialogblockaden. Linguistische und politikwissenschaftliche Studien zur politischen Kommunikation. Berlin, S. 3-30.

Klein, Josef (1989): Wortschatz, Wortkampf, Wortfelder in der Politik. In: Klein, Josef (Hg.): Politische Semantik. Beträge zur politischen Sprachverwendung, Opladen. 
Klein, Josef (2000): Textsorten im Bereich politischer Institutionen. In: Brinker, Klaus et al. (Hg.): Text- und Gesprächslinguistik. Ein internationales Handbuch zeitgenössischer Forschung. Berlin, S. 732-755.

Klinker, Fabian/Scharloth, Joachim/Szczęk, Joanna (Hg.) (2018): Sprachliche Gewalt. Formen und Effekte von Pejorisierung, verbaler Aggression und Hassrede. Stuttgart.

Koch, Peter/Oesterreicher, Wulf (2008): Mündlichkeit und Schriftlichkeit von Texten. In: Janich, Nina (Hg.): Textlinguistik. 15 Einführungen. Tübingen, S. 199-216.

Löffler, Miriam (2014): Think Content! Content-Strategie, Content-Marketing, Texten fürs Web. Bonn.

Makowski, Jacek (2011a): Zum Wesen der Sprachmanipulation. In: Makowski, Jacek (Hg.): How not to Do Things with Words: Beiträge zur Sprache in Politik, Recht und Werbung. Łódź.

Makowski, Jacek (Hg.) (2011b): How not to Do Things with Words: Beiträge zur Sprache in Politik, Recht und Werbung. Łódź.

Makowski, Jacek (2013): Die Abgeordnetenrede im Europäischen Parlament: Korpusgestützte textsortenorientierte Analyse deutschsprachiger Wortmeldungen in den Plenardebatten des Europäischen Parlaments. Łódź.

Meibauer, Jörg (2013): Hassrede - von der Sprache zur Politik. In: Meibauer, Jörg (Hg.): Hassrede/Hate Speech. Interdisziplinäre Beiträge zu einer aktuellen Diskussion. Gießen, S. 1-16. Quelle: geb.uni-giessen.de/geb/volltexte/2013/9251 (01.12.2018).

Merten, Klaus (2000): Das Handwörterbuch der PR. 2 Bde. Frankfurt am Main.

Merten, Klaus (2008): Kommunikation und Persuasion. In: Bentele, Günter/ Fröhlich, Romy/Szyszka, Peter (Hg.): Handbuch der Public Relations. Wissenschaftliche Grundlagen und berufliches Handeln. Wiesbaden, S. 297308.

Mikołajczyk, Beata (2004): Sprachliche Mechanismen der Persuasion in der politischen Kommunikation. Dargestellt an polnischen und deutschen Texten zum EU-Beitritt Polens. Frankfurt am Main.

Ortak, Nuri (2004): Persuasion. Zur textlinguistischen Beschreibung eines dialogischen Strategiemusters. Tübingen.

Pappert, Steffen (2017): Plakatbusting: Die Umwandlung von Wahlplakaten in transgressive Sehflächen. In: Kämper, Heidrun/Wengeler, Martin (Hg.): Protest - Parteischelte - Politikverdrossenheit: Politikkritik in der Demokratie. Bremen, S. 55-76.

Reisigl, Martin (2011): Kommunikationstypologien des Handlungsbereiches Politik. In: Habscheid, Stefan (Hg.): Textsorten und sprachliche Handlungsmuster: Linguistische Typologien der Kommunikation. Berlin, S. 437-472.

Reissen-Kosch, Jana (2016): Identifikationsangebote der rechten Szene im Netz. Linguistische Analyse persuasiver Online-Kommunikation. Bremen. 
Römer, Christine (2012): Werbekommunikation lexikologisch. In: Janich, Nina (Hg.): Handbuch Werbekommunikation. Sprachwissenschaftliche und interdisziplinäre Zugänge. Tübingen, S. 33-47.

Römer, Ruth (1980): Die Sprache der Anzeigenwerbung. 6. Aufl. Düsseldorf.

Roth, Reinhold (1987): Parteimanagement und politische Führungseliten in der Politikvermittlung. In: Sarcinelli, Ulrich (Hg.): Politikvermittlung. Beiträge zur politischen Kommunikationskultur. Stuttgart, S. 184-202.

Sarcinelli, Ulrich (2005): Politische Kommunikation in Deutschland. Zur Politikvermittlung im demokratischen System. Wiesbaden.

Sarcinelli, Ulrich (2009): Politische Kommunikation in Deutschland. Zur Politikvermittlung im demokratischen System. 2., überarbeitete und erweiterte Auflage. Wiesbaden.

Schach, Annika (2015): Advertorial, Blogbeitrag, Content-Strategie \& Co. Neue Texte der Unternehmenskommunikation. Wiesbaden.

Schröter, Melani/Carius, Björn (2009): Vom politischen Gebrauch der Sprache. Wort, Text, Diskurs. Eine Einführung. Frankfurt am Main.

Schweiger, Günter/Schrattenecker, Gertraud (1995): Werbung. Eine Einführung. 4., völlig neu bearbeitete und erweiterte Auflage. Stuttgart/Jena.

Schweiger, Wolfgang/Jungnickel, Katrin (2011): Pressemitteilungen 2.0 - eine Resonanzanalyse im Internet. In: Publizistik 4, S. 399-421.

Siever, Torsten/Schlobinski, Peter (Hg.) (2012): Entwicklungen im Web 2.0. Ergebnisse des III. Workshops zur linguistischen Internetforschung. Band 3. Frankfurt am Main.

Simmler, Franz (1998): Fachsprachliche Phänomene in den öffentlichen Texten von Politikern. In: Hoffmann, Lothar/Kalverkämper, Hartwig/Wiegand, Herbert Ernst (Hg.): Fachsprachen. Ein internationales Handbuch zur Fachsprachenforschung und Terminologiewissenschaft. Berlin, S. 736-756.

Solis, Brian/Breakenridge, Deirdre (2010): Putting the Public Back in Public Relations: How Social Media Is Reinventing the Aging Business of PR. New Jersey.

Stöckl, Hartmut (1997): Werbung in Wort und Bild: Textstil und Semiotik englischsprachiger Anzeigenwerbung. Frankfurt am Main.

Tillmann, Alexander (1989): Ausgewählte Textsorten politischer Sprache. Eine linguistische Analyse parteilichen Sprechens. Göppingen.

White, Candace/Raman, Niranjan (1999): The world wide web as a public relations medium: The use of research, planning, and evaluation in web site development. In: Public Relations Review 25, S. 405-419.

Wolff, Uwe (2010): Medienarbeit für Rechtsanwälte. Ein Handbuch für effektive Kanzlei-PR. Wiesbaden.

Woźniak-Łojczuk, Elżbieta (2010): Komunikat prasowy jako podstawowe narzędzie w działaniach media relations uczelni wyższych: Politechniki Wrocławskiej, Uniwersytetu Ekonomicznego, Uniwersytetu Przyrodnicze- 
go oraz Uniwersytetu Wrocławskiego. In: Borkowski, Igor/Stasiuk-Krajewska, Karina (Hg.): Dziennikarstwo i media: Przestrzenie komunikowania. Wrocław, S. 185-209.

Zimmermann, Hans Dieter (1969): Die politische Rede. Der Sprachgebrauch Bonner Politiker. Stuttgart/Berlin /Köln/Mainz.

Zehrt, Wolfgang (2014): Die Pressemitteilung. 2., völlig überarbeitete Auflage. Konstanz.

\section{Od kreowania wizerunku do dyskredytacji przeciwników politycznych. Mowa nienawiści jako strategia w komunikatach prasowych}

Abstrakt: Gdy w komunikatach prasowych zamieszczonych na oficjalnym portalu partii Alternatywa dla Niemiec (AfD) odnajdujemy w odniesieniu do konkurencyjnych ugrupowań i polityków określenia takie jak czerwony pokaz odmieńców (socjaldemokraci) czy sadystyczna domina (Angela Merkel), nasuwa się pytanie o cel, w jakim elementy mowy nienawiści znajdują zastosowanie w gatunku tekstu, którego prototypicznie nadrzędną funkcją jest przekazywanie informacji o wartości medialnej oraz pozytywne nacechowanie nadawcy tekstu. Przedmiotem niniejszych rozważań jest mowa nienawiści w komunikatach prasowych partii politycznych jak strategia dyskredytacji przeciwników politycznych. Wychodząc od omówienia cech gatunkowych komunikatu prasowego, podjęta zostanie próba określenia jego funkcji w obszarze komunikacji politycznej z punktu widzenia typologii tekstów i gatunków (rodzajów) tekstów politycznych. W dalszej części analizie ilościowej poddany zostanie wyekscerpowany materiał w postaci sześćdziesięciu komunikatów prasowych partii Alternatywa dla Niemiec oraz przeprowadzona zostanie analiza jakościowa jednego wybranego tekstu komunikatu ze szczególnym uwzględnieniem przejawów mowy nienawiści na płaszczyźnie pragmatycznej, semantycznej, gramatycznej oraz retorycznej.

Słowa kluczowe: komunikat prasowy online, mowa nienawiści, gatunek tekstu politycznego, dyskredytacja, język polityki.

\section{Frome Image Building to Discrediting Political Opponents. Hate Speech as a Strategy in Press Releases}

Abstract: The subject of these considerations is hate speech in press releases of political parties as a strategy to discredit political opponents. The article published on the official website of the Alternative for Germany (AfD) contained terms 
such as the red show of misfits (social democrats) or sadistic domino (Angela Merkel) in relation to competing groups and politicians. The question arises as to the purpose of using such elements in types of texts whose primary function is to transmit information in a positive way and as a result relay the writer's optimism. The paper shall start from the presentation of specific features of press releases; furthermore, an attempt will be made to determine its function in the field of political communication from the point of view of political text typology and genres. Throughout the rest of the paper quantitative analysis of selected materials will be presented, including sixty press releases of the Alternative for Germany party and a qualitative analysis of one selected text will be conducted. Special emphasis will be put on the pragmatic, semantic, grammatical and rhetorical levels of hate speech.

Key words: online press release, hate speech, political genre, discrediting, language of politics. 


\section{Untersuchungskorpus}

\section{Alternative für Deutschland, Online-Pressemitteilungen, Februar 2018.}

Tab. 1

Alternative für Deutschland, Online-Pressemitteilungen, Februar 2018

\begin{tabular}{|c|l|l|}
\hline Nr. & \multicolumn{1}{|c|}{ Titel } & Orts- und Zeitangabe \\
\hline T01 & $\begin{array}{l}\text { Georg Pazderski: Syrien jetzt als sicheres Herkunftsland } \\
\text { einstufen }\end{array}$ & Berlin, 1. Februar 2018 \\
\hline T02 & $\begin{array}{l}\text { Jörg Meuthen: Kein Familiennachzug für subsidiär } \\
\text { Schutzberechtigte }\end{array}$ & Berlin, 1. Februar 2018 \\
\hline T03 & $\begin{array}{l}\text { Uwe Schulz: CDU übernimmt AfD-Forderung nach ge- } \\
\text { samtgesellschaftlicher Diskussion über die Digitalisierung }\end{array}$ & Berlin, 1. Februar 2018 \\
\hline T04 & $\begin{array}{l}\text { Stephan Brandner: Schluss mit steuerfinanzierten Mig- } \\
\text { rantenflügen }\end{array}$ & Berlin, 1. Februar 2018 \\
\hline T05 & $\begin{array}{l}\text { Hansjörg Müller: Die Strategie der Hinterzimmer geht } \\
\text { auf - Mercosur ist der nächste große Deal }\end{array}$ & Berlin, 1. Februar 2018 \\
\hline T06 & $\begin{array}{l}\text { Georg Pazderski: Dem Treiben der ideologieverblendeten } \\
\text { Gutmenschen Einhalt gebieten }\end{array}$ & Berlin, 2. Februar 2018 \\
\hline T07 & $\begin{array}{l}\text { Georg Pazderski: Wehrpflicht wieder einsetzen, Verteidi- } \\
\text { gungsetat deutlich erhöhen }\end{array}$ & Berlin, 2. Februar 2018 \\
\hline T08 & Markus Frohnmaier: Keuschheitsgürtel für Frauen & Berlin, 2. Februar 2018 \\
\hline T09 & $\begin{array}{l}\text { Bernd Baumann: Linksextreme Randalierer und Plünde- } \\
\text { rer konsequent verfolgen }\end{array}$ & Berlin, 2. Februar 2018 \\
\hline T10 & $\begin{array}{l}\text { Israelische Geheimdienstlegende Rafi Eitan unterstützt } \\
\text { AfD }\end{array}$ & Berlin, 2. Februar 2018 \\
\hline T11 & Robby Schlund: Kinderlachen statt Abtreibung & Berlin, 2. Februar 2018 \\
\hline T12 & $\begin{array}{l}\text { Alice Weidel: Alarmierende Zahl von Angriffen auf } \\
\text { Christen verlangt Taten statt Sprüche }\end{array}$ & Berlin, 2. Februar 2018 \\
\hline T13 & $\begin{array}{l}\text { Anton Friesen: Zum 25-jährigen Jubiläum der } \\
\text { deutsch-aserbaidschanischen Beziehungen }\end{array}$ & Berlin, 5. Februar 2018 \\
\hline T14 & $\begin{array}{l}\text { Lars Herrmann: Abschiebungen auch in Deutschland } \\
\text { forcieren }\end{array}$ & Berlin, 5. Februar 2018 \\
\hline
\end{tabular}


Tab. 1

\begin{tabular}{|c|c|c|}
\hline Nr. & Titel & Orts- und Zeitangabe \\
\hline T15 & $\begin{array}{l}\text { Uwe Kamann: OECD-Zahlen belegen - Deutsche fallen } \\
\text { ins Rentenloch }\end{array}$ & Berlin, 5. Februar 2018 \\
\hline T16 & $\begin{array}{l}\text { Georg Pazderski: Israel macht es vor - Afrikanische } \\
\text { Migranten werden ausgewiesen }\end{array}$ & Berlin, 5. Februar 2018 \\
\hline T17 & $\begin{array}{l}\text { Alice Weidel: GroKo treibt die Leistungsträger der Mittel- } \\
\text { schicht in die Resignation und aus dem Land }\end{array}$ & Berlin, 5. Februar 2018 \\
\hline T18 & $\begin{array}{l}\text { Stephan Brandner: Steigende Zahl deutscher Gefangener } \\
\text { in Türkei ist besorgniserregend }\end{array}$ & Berlin, 6. Februar 2018 \\
\hline T19 & $\begin{array}{l}\text { Götz Frömming: Aufhebung des „Kooperationsverbots“ } \\
\text { ist teure Schaufensterpolitik }\end{array}$ & Berlin, 6. Februar 2018 \\
\hline $\mathrm{T} 20$ & Paul Hampel: Nichts geht mehr & Berlin, 7. Februar 2018 \\
\hline $\mathrm{T} 21$ & $\begin{array}{l}\text { Stephan Brandner: Rundfunkbeitragswahnsinn - Allein } \\
\text { der Bund zahlte } 2017 \text { über 1,5 Millionen Euro Rundfunk- } \\
\text { beiträge }\end{array}$ & Berlin, 7. Februar 2018 \\
\hline $\mathrm{T} 22$ & $\begin{array}{l}\text { Peter Felser informiert sich vor Ort bei der Bundespolizei } \\
\text { in Kempten über illegale Migration und Sozialbetrug } \\
\text { durch Zuwanderer }\end{array}$ & Berlin, 8. Februar 2018 \\
\hline $\mathrm{T} 23$ & $\begin{array}{l}\text { Leif-Erik Holm: GroKo setzt energiepolitische Geister- } \\
\text { fahrt fort }\end{array}$ & Berlin, 8. Februar 2018 \\
\hline $\mathrm{T} 24$ & $\begin{array}{l}\text { Rüdiger Lucassen: Von der Leyen bleibt Verteidigungsmi- } \\
\text { nisterin - GroKo nimmt auf Bundeswehr keine Rücksicht }\end{array}$ & Berlin, 7. Februar 2018 \\
\hline $\mathrm{T} 25$ & $\begin{array}{l}\text { Georg Pazderski: So sieht Fürsorge unter der designierten } \\
\text { Verteidigungsministerin von der Leyen aus }\end{array}$ & Berlin, 8. Februar 2018 \\
\hline T26 & $\begin{array}{l}\text { Tino Chrupalla: Im Jahreswirtschaftsbericht der Bundes- } \\
\text { regierung wird das Handwerk nicht erwähnt }\end{array}$ & Berlin, 8. Februar 2018 \\
\hline $\mathrm{T} 27$ & Uwe Kamann: Heiße Luft statt Digitalisierungsstrategie & Berlin, 9. Februar 2018 \\
\hline $\mathrm{T} 28$ & $\begin{array}{l}\text { Enrico Komning: Koalitionsvertrag wirft Deutschland } \\
\text { weiter zurück }\end{array}$ & Berlin, 9. Februar 2018 \\
\hline T29 & $\begin{array}{l}\text { Jochen Haug: Im Koalitionsvertrag fehlen die Signale für } \\
\text { eine migrationspolitische Wende }\end{array}$ & Berlin, 9. Februar 2018 \\
\hline T30 & Jürgen Pohl: GroKo überlässt Ostdeutschland der Armut & Berlin, 9. Februar 2018 \\
\hline T31 & Markus Frohnmaier: Vorsicht vor Fake-News über Syrien & Berlin, 9. Februar 2018 \\
\hline T32 & $\begin{array}{l}\text { Marc Jongen: Das als „Geschlechtergerechtigkeit“ getarn- } \\
\text { te Quotenunwesen muss beendet werden }\end{array}$ & Berlin, 9. Februar 2018 \\
\hline
\end{tabular}




\begin{tabular}{|c|c|c|}
\hline T33 & $\begin{array}{l}\text { Siegbert Droese: Koalitionsvertrag gefährdet Deutsch- } \\
\text { lands Souveränität }\end{array}$ & Berlin, 9. Februar 2018 \\
\hline T34 & $\begin{array}{l}\text { Alice Weidel: EU-Kommission schadet mit Bestrafungs- } \\
\text { mechanismus für Großbritannien der europäischen } \\
\text { Wirtschaft }\end{array}$ & Berlin, 9. Februar 2018 \\
\hline T35 & $\begin{array}{l}\text { Alexander Gauland: Der politisch-mediale Komplex } \\
\text { beschädigt die Glaubwürdigkeit der deutschen Medien- } \\
\text { landschaft }\end{array}$ & Berlin, 9. Januar 2018 \\
\hline T36 & Alice Weidel: Schulz‘ Abgang sollte erst der Anfang sein & Berlin, 9. Februar 2018 \\
\hline T37 & $\begin{array}{l}\text { Georg Pazderski: Bundeswehr konsolidieren, statt Aus- } \\
\text { landseinsätze ausweiten }\end{array}$ & $\begin{array}{l}\text { Berlin, 12. Februar } \\
2018\end{array}$ \\
\hline T38 & $\begin{array}{l}\text { Weidel/Gauland: Die AfD wird Merkel den Ausverkauf } \\
\text { von Deutschlands Zukunft nicht durchgehen lassen }\end{array}$ & $\begin{array}{l}\text { Berlin, 12. Februar } \\
2018\end{array}$ \\
\hline T39 & $\begin{array}{l}\text { Jörg Meuthen und Harald Vilimsky sprechen beim Politi- } \\
\text { schen Aschermittwoch der AfD }\end{array}$ & $\begin{array}{l}\text { Berlin, 12. Februar } \\
2018\end{array}$ \\
\hline $\mathrm{T} 40$ & Alexander Gauland: Österreich macht es vor & $\begin{array}{l}\text { Berlin, 13. Februar } \\
2018\end{array}$ \\
\hline T41 & $\begin{array}{l}\text { Georg Pazderski: Vertrauensverlust der Deutschen in die } \\
\text { Bundeswehr ist alarmierend }\end{array}$ & $\begin{array}{l}\text { Berlin, 15. Februar } \\
2018\end{array}$ \\
\hline $\mathrm{T} 42$ & $\begin{array}{l}\text { Alexander Gauland: Schluss mit zwangsfinanzierten } \\
\text { Propagandafantasien }\end{array}$ & $\begin{array}{l}\text { Berlin, 15. Februar } \\
2018\end{array}$ \\
\hline T43 & $\begin{array}{l}\text { Jörg Meuthen: Rund } 1.000 \text { Besucher beim Politischen } \\
\text { Aschermittwoch der AfD }\end{array}$ & $\begin{array}{l}\text { Osterhofen, 16. Febru- } \\
\text { ar } 2018\end{array}$ \\
\hline $\mathrm{T} 44$ & $\begin{array}{l}\text { Alice Weidel: Nach Brexit - EU-Haushalt kürzen, statt } \\
\text { deutsche Steuerzahler noch weiter schröpfen }\end{array}$ & $\begin{array}{l}\text { Berlin, 16. Februar } \\
2018\end{array}$ \\
\hline $\mathrm{T} 45$ & $\begin{array}{l}\text { Jörg Meuthen: Mehr Bürgernähe wird nicht durch mehr } \\
\text { Machtkonzentration in Brüssel erreicht }\end{array}$ & $\begin{array}{l}\text { Berlin, 16. Februar } \\
2018\end{array}$ \\
\hline T46 & $\begin{array}{l}\text { Georg Pazderski: Soll von der Leyen nach der Bundes- } \\
\text { wehr nun die NATO ruinieren? }\end{array}$ & $\begin{array}{l}\text { Berlin, 19. Februar } \\
2018\end{array}$ \\
\hline T47 & $\begin{array}{l}\text { Alexander Gauland: Einzig die deutsche Regierung han- } \\
\text { delt gegen die Interessen der eigenen Bürger }\end{array}$ & $\begin{array}{l}\text { Berlin, 20. Februar } \\
2018\end{array}$ \\
\hline $\mathrm{T} 48$ & $\begin{array}{l}\text { Georg Pazderski: Probleme der Bundeswehr lösen, statt } \\
\text { immer neue Auslandseinsätze }\end{array}$ & $\begin{array}{l}\text { Berlin, 20. Februar } \\
2018\end{array}$ \\
\hline T49 & Alexander Gauland: Endlich konsequent abschieben & $\begin{array}{l}\text { Berlin, 20. Februar } \\
2018\end{array}$ \\
\hline $\mathrm{T} 50$ & $\begin{array}{l}\text { Jörg Meuthen: EU institutionell konsolidieren, statt im- } \\
\text { mer neue Mitglieder aufnehmen }\end{array}$ & $\begin{array}{l}\text { Berlin, 22. Februar } \\
2018\end{array}$ \\
\hline
\end{tabular}


Tab. 1

\begin{tabular}{|c|l|l|}
\hline Nr. & \multicolumn{1}{|c|}{ Titel } & Orts- und Zeitangabe \\
\hline T51 & $\begin{array}{l}\text { Alexander Gauland: Polen und andere Mitgliedsstaaten } \\
\text { dürfen sich nicht von Merkel erpressen lassen }\end{array}$ & $\begin{array}{l}\text { Berlin, 23. Februar } \\
2018\end{array}$ \\
\hline T52 & $\begin{array}{l}\text { Alice Weidel kritisiert „Zwei-Klassen-Sozialstaat zum } \\
\text { Nachteil Einheimischer“ }\end{array}$ & $\begin{array}{l}\text { Berlin, 23. Februar } \\
2018\end{array}$ \\
\hline T53 & $\begin{array}{l}\text { Meuthen/Felser: AfD fordert ersatzlosen Wegfall der } \\
\text { britischen Sitze im EU-Parlament nach dem „Brexit“ }\end{array}$ & $\begin{array}{l}\text { Berlin, 23. Februar } \\
2018\end{array}$ \\
\hline T54 & $\begin{array}{l}\text { Jörg Meuthen: Merkels letztes Aufgebot } \\
\text { Berlin, 25. Februar } \\
2018\end{array}$ \\
\hline T55 & $\begin{array}{l}\text { Alice Weidel: Barleys Kritik an der „Tafel Essen“ offen- } \\
\text { bart völlige Verantwortungslosigkeit }\end{array}$ & $\begin{array}{l}\text { Berlin, 26. Februar } \\
2018\end{array}$ \\
\hline T56 & $\begin{array}{l}\text { Georg Pazderski: Nochmal vier Jahre von der Leyen sind } \\
\text { eine Katastrophe für die Bundeswehr }\end{array}$ & $\begin{array}{l}\text { Berlin, 26. Februar } \\
2018\end{array}$ \\
\hline T57 & $\begin{array}{l}\text { Georg Pazderski: Merkel braucht Spahn als konservatives } \\
\text { Feigenblatt }\end{array}$ & $\begin{array}{l}\text { Berlin, 27. Februar } \\
2018\end{array}$ \\
\hline T58 & $\begin{array}{l}\text { Gauland/Meuthen: Gott sei Dank gibt es noch Richter in } \\
\text { Karlsruhe }\end{array}$ & $\begin{array}{l}\text { Berlin, 27. Februar } \\
2018\end{array}$ \\
\hline T59 & $\begin{array}{l}\text { Kay Gottschalk: Kramp-Karrenbauer gleich Stillstand für } \\
\text { Deutschland }\end{array}$ & $\begin{array}{l}\text { Berlin, 27. Februar } \\
2018\end{array}$ \\
\hline T60 & $\begin{array}{l}\text { Weidel/Gauland: Das Kooperationsverbot mit der AfD } \\
\text { zeugt von absoluter Inhaltsleere der Union }\end{array}$ & $\begin{array}{l}\text { Berlin, 28. Februar } \\
2018\end{array}$ \\
\hline
\end{tabular}

Quelle: https://www.afd.de/2018/02. Breadcrumb: https://www.afd.de $\rightarrow$ Presse $\rightarrow$ Pressemitteilungen $\rightarrow$ Archiv $\rightarrow$ Februar 2018 (60). Abgerufen am 14. März 2018. 


\section{Textbeispiel (T43)}

Jörg Meuthen: Rund 1.000 Besucher beim Politischen Aschermittwoch der AfD

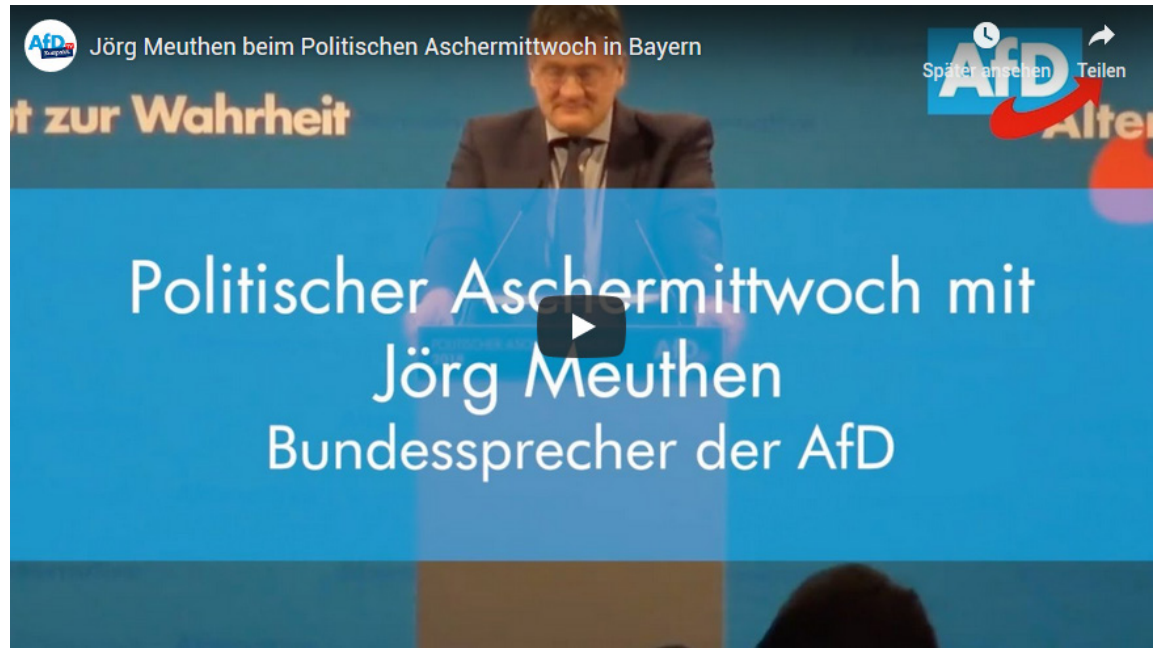

Osterhofen, 16. Februar 2018. Die zerstrittene und in der Wählergunst abstürzende SPD wird bald mehr Parteimitglieder, als Wähler haben und bei der CSU symbolisiert der Buchstabe „C" inzwischen die islamische Halbmond-Sichel anstelle des Christentums. Für zugespitzte Aussagen wie diese bekam der AfD-Bundesvorsitzende Jörg Meuthen beim Politischen Aschermittwoch in Osterhofen stürmischen Applaus.

Mehr als 1.000 Gäste waren in die voll besetzte Halle des „Donaucenter Schubert“ gekommen, um ihn zu hören sowie Bayerns Landeschef Martin Sichert, seine Stellvertreterin im Landesvorstand Katrin Ebner-Steiner und als Gastredner Harald Vilimsky, Generalsekretär der österreichischen FPÖ. Es war der erste Politische Aschermittwoch der AfD seit ihrem Einzug als drittstärkste Kraft in den Deutschen Bundestag mit 92 Abgeordneten, von denen 14 aus Bayern kommen. So auch der niederbayerische Bezirksvorsitzende Stephan Protschka, der als Moderator durch das mehrstündige Programm führte.

Mit der Großveranstaltung wurde zugleich auf den bayerischen Landtagswahlkampf eingestimmt. „Die AfD wird im nächsten Landtag sitzen und 
eine bessere Politik machen, als die CSU“, rief Meuthen unter starkem Beifall in die Halle. Am 14. Oktober wird Bayern gewählt und zwei Wochen später in Hessen. Danach wird die AfD nach einer ununterbrochenen Siegesserie in allen 16 Landtagen mit schätzungsweise 200 Abgeordneten vertreten sein.

Rund 30 Medienvertreter waren nach Osterhofen gereist. Alle großen Fernsehsender berichteten zum Teil live mit Interviews aus der mit weiß-blauen Tischdecken und AfD-Plakaten dekorierten Halle. Blasmusik, Brezeln, Weißwürste sowie Dirndl und Trachten der Besucher unterstrichen die Volksfeststimmung an den langen Biertischen. Vor dem Donaucenter hatte die in überschaubarer Zahl präsente Polizei wenig zu tun. Die Linke konnte nur ein Dutzend kleinlauter Gegendemonstranten am Straßenrand aufbieten.

„Es heißt, am Aschermittwoch ist alles vorbei“, rief Meuthen in Erinnerung und fügte genüsslich hinzu: „Für manche in der Politik stimmt das tatsächlich, etwa für Martin Schulz." Der gescheiterte Hoffnungsträger, von den eigenen Genossen gestoppte Außenminister-Kandidat und nach nur einem Jahr vom Amt zurückgetretene SPD-Parteichef werde der geplanten "Groko-Gruselkoalition" nicht in der Regierung angehören, freute sich Meuthen. Als „rote Freak-Show“ bezeichnete er den gegen die AfD pöbelnden SPD-Parteivize Ralf Stegner „der bald über seine eigenen heruntergezogenen Mundwinkel stolpern wird“, das „Maas-Männchen“ Bundesjustizminister Heiko Maas und die zuweilen im Bundestag singende Fraktionschefin Andrea Nahles. Tosenden Applaus und Bravo-Rufe gab es zu Meuthens Hinweis, es sei heutzutage offenbar „ein Malus“ Deutscher zu sein, weil Asylbewerber und Migranten mehr soziale Unterstützung bekämen, als Einheimische.

In der „Noch-Kanzlerin und Noch-CDU-Vorsitzenden“ Angela Merkel sieht der AfD-Parteichef weniger die viel zitierte „Mutti“, als vielmehr eine sadistische Domina, der sich auch CSU-Chef „Heißluft-Horst Seehofer" als künftiger Bundesinnenminister devot unterordne. Obwohl Merkel als Rednerin „die personifizierte Schlaftablette“ sei, applaudiere man ihr bei Parteitagen artig wie die „Duracell-Klatschhasen“, schilderte Meuthen kopfschüttelnd zum Niedergang dieser einst großen konservativen Partei. $\mathrm{Zu}$ den lang anhaltenden lautstarken „Merkel muss weg“-Sprechchören der 1.000 Aschermittwochs-Besucher stellte er fest: „Das ist nicht nur die 
Stimmung hier im Festzelt, sondern in ganz Deutschland." Neben SPD und Union bekamen auch die kleineren Parteien ihr Fett weg. Grüne und Linke seien nur "politische Nischen-Anbieter", sagte Meuthen. Die selbstverliebte "Christian-Lindner-Partei“ wiederum sei nur ,älteren Semestern auch noch als FDP bekannt". Für die CSU sei längst charakteristisch, dass zwischen Reden und Handeln ein maximaler Widerspruch bestehe.

FPÖ-Gast Vilimsky, wie Meuthen Abgeordneter im Europaparlament, betonte die Gemeinsamkeiten mit der AfD in der Europapolitik und dass seine Partei mit der Regierungsbeteiligung in Österreich eine Wende in der Asylpolitik erzwungen habe. FPO-Chef Heinz-Christian Strache wurde dort Vizekanzler. Er war im Vorjahr AfD-Gastredner in Osterhofen. Was der FPÖ im Nachbarland gelungen sei, könne die AfD auch in Deutschland erreichen. „Die Zukunft gehört uns, wenn wir uns selbst treu bleiben“, gab Vilimsky als Ratschlag unter Beifall mit auf den Weg.

Der AfD-Landeschef und Bundestagsabgeordnete Martin Sichert prangerte einen „Rassismus gegen die eigene Bevölkerung“ an, weil untätige Asylbewerber in vielen Bereichen besser unterstützt würden, als die Einheimischen. Während Entbindungsstationen oder Krankenhausplätze aus Kostengründen massiv abgebaut würden, „schossen Asylbewerberheime wie Pilze aus dem Boden“, kritisierte er. „Statt einer Willkommenskultur für illegale Einwanderer brauchen wir eine Willkommenskultur für Kinder", forderte Sichert unter Applaus. Mit Blick auf die Landtagswahl fügte er hinzu: „Mit der Dampfplauderei der CSU ist bald Schluss, die werden ihr blaues Wunder erleben." Seehofer sei dank der AfD bereits weg aus Bayern. „Und wenn die SPD sich selbst zerlegt, dann ist es zum ersten Mal, dass sie etwas sinnvolles für unser Land tut", lobte der Landeschef ironisch. Den Grünen warf er „eine militante Energiepolitik“ vor und vermeidbaren Flächenfraß durch Abholzung für immer mehr Windräder samt betonierter Zufahrtswege.

Vize-Landesvorsitzende Ebner-Steiner warf der CSU wegen Moschee-Neubauten eine Islamisierung Bayerns vor und kritisierte: „Das ist Leitkultur Allah-CSU.“ Sie möchte weiterhin lieber Miniröcke statt Burka sehen. „In Bayern gehört der Islam nicht zu Deutschland und nur mit uns bleibt die Kirche im Dorf", rief sie unter lautem Beifall in den Saal. Die AfD habe schon bei der Bundestagswahl in Bayern das beste westdeutsche Ergebnis geholt. In ihrem Wahlkreis erzielte sie fast 20 Prozent und machte 
Deggendorf zur AfD-Hochburg. Angesichts zweistelliger Prognosen für die AfD zur Landtagswahl, habe „die CSU die Lederhosen gestrichen voll“ und Seehofer habe bereits „als Flüchtling in Merkels Regierung Asyl gefunden". Allerdings nur subsidiär und befristet auf maximal vier Jahre bis zur nächsten Bundestagswahl.

Sein Nachrücker-Ministerpräsident Markus Söder werde die in der Wählergunst sinkende CSU, „diese kleine regionale Voralpen-Partei“, auch nicht mehr retten. „Denn wir ziehen auf jeden Fall in den Landtag ein, das ist so sicher wie das Amen in der Kirche", betonte Ebner-Steiner und erklärte die Erfolge der AfD ganz einfach: „Die Altparteien haben den Klimawandel komplett verpennt, nicht den am Nordpol, sondern den gesellschaftlichen und politischen Klimawandel in Deutschland und in Bayern."

Pressemitteilung Jörg Meuthen: Rund 1.000 Besucher beim Politischen Aschermittwoch der AfD vom 16. Februar 2018. Quelle: www.afd.de/joerg-meuthen-rund-1-000-besucher-beim-politischen-aschermittwoch-der-afd/ (23.11.2018).

Verlinkung zum Video: Jörg Meuthen beim Politischen Aschermittwoch in Bayern vom 14. Februar 2018. Quelle: AfD Kompakt TV, www.youtube.com/watch?time_continue $=6 \& v=F M Q 1 C N a C P Y E ~(23.11 .2018)$. 